

\title{
Theoretical analysis of damping effects of guided elastic waves at solid/fluid interfaces
}

\author{
Sylvain Ballandras, Alexandre Reinhardt, Abdelkrim Khelif, Mikael Wilm, \\ Vincent Laude, William Daniau, Virginie Blondeau-Patissier
}

\section{To cite this version:}

Sylvain Ballandras, Alexandre Reinhardt, Abdelkrim Khelif, Mikael Wilm, Vincent Laude, et al.. Theoretical analysis of damping effects of guided elastic waves at solid/fluid interfaces. Journal of Applied Physics, 2006, 99, pp.054907. 10.1063/1.2168242 . hal-00073046

\section{HAL Id: hal-00073046 https://hal.science/hal-00073046}

Submitted on 24 May 2006

HAL is a multi-disciplinary open access archive for the deposit and dissemination of scientific research documents, whether they are published or not. The documents may come from teaching and research institutions in France or abroad, or from public or private research centers.
L'archive ouverte pluridisciplinaire HAL, est destinée au dépôt et à la diffusion de documents scientifiques de niveau recherche, publiés ou non, émanant des établissements d'enseignement et de recherche français ou étrangers, des laboratoires publics ou privés. 


\title{
Theoretical analysis of damping effects of guided elastic waves at solid/fluid interfaces
}

\author{
S. Ballandras, A. Reinhardt, A. Khelif, M. Wilm, V. Laude, W. Daniau, V. Blondeau-Pâtissier
}

Institut FEMTO-ST, UMR CNRS-UFC-ENSMM-UTBM 6174,

Département LPMO, 32 Avenue de l'Observatoire, F-25044 Besançon Cedex

\begin{abstract}
A theoretical description of ideal and viscous fluid media is proposed to address the problem of modelling damping effects of Surface Acoustic Waves (SAW) and more generally of any guided elastic waves at the interface between viscous fluids and solids. It is based on the Fahmy-Adler eigenvalue representation of the elastic propagation problem, extended to provide the Green's function of the considered media. It takes advantage of previous efforts developed to numerically stabilize the Green's function computation process. This function is used to compute an harmonic admittance according to the Blötekjaër approach. The influence of acoustic radiation and viscosity effects on different kind of waves excited on various substrates is reported and discussed.
\end{abstract}

\section{Introduction}

Surface Acoustic Waves (SAW) can be excited at the surface of any solid material. These waves may exhibit elliptic as well as pure shear polarisation (case of isotropic media), but the practical case of wave propagation at the surface of anisotropic material generally yields any combination of wave polarisation, except along given crystal or symmetry axes [1]. True SAW are assumed to propagate without any losses along the guiding surface, providing a nice opportunity to manufacture low loss devices such as filters and resonators. However, it is also known that best quality factors and/or smallest insertion losses of SAW devices are obtained using package closed under vacuum to avoid leakage due to acoustic radiation in air. The use of SAW devices for the development of sensors immersed in fluid media also has been widely investigated. For instance, Rayleigh waves are known to be dramatically damped by water but pure shear waves are often considered capable to exist even when their propagation substrate is loaded by a liquid. Theoretical analysis of SAW excitation and propagation under such working conditions requires the adaptation of existing simulation tools to provide a reliable description of the induced effects by the nature of the surrounding medium on the SAW device response. 
In the proposed paper, the way the Green's function analysis and the harmonic admittance can be used in that matter is described. The mathematical developments required to develop a computation tool based on such concepts are exposed. The case of viscous fluids (in the limit of the Newtonian fluid assumption [2]) has been particularly investigated, since it also allows to simulate ideal fluids simply by setting the viscosity coefficient to zero. Many cases then are considered to illustrate the interest of the proposed approach, for instance the attenuation of Rayleigh waves due to water damping, the sensitivity of Leaky waves to viscosity or the behaviour of plate modes loaded on one side by viscous water. The theoretical results are then discussed. The specific situation of pure shear waves as used in surface transverse waves (STW) is particularly regarded. These waves are generally assumed poorly affected by water and other weakly viscous fluids. The limit of this hypothesis is examined theoretically.

\section{Theoretical fundaments}

II.1 Modelling the acoustic behaviour of ideal and viscous fluids

The theoretical representation of acoustic waves in fluids is usually performed using a pressure formulation. Nevertheless, in order to easily derive the corresponding Green's function, a displacement formulation can be constructed as well. For any fluid, the independent elastic constants required for such a formulation reduce to one, i.e. $C_{11}$ which is also equal to $C_{12}$, yielding $C_{66}=0$ consequently. According to [2], a shear effect in a fluid between a moving solid and a reference solid results in a linear stress proportional to the velocity gradient via a coefficient written $\eta$ called shear viscosity or absolute viscosity of the fluid. In an isotropic homogeneous uncompressible Newtonian fluid, the stress is proportional to the linear strain ; this is called the Stokes low. In a very general approach [3], one should also consider a compressive viscosity factor written $\zeta$. The pressure in the fluid $P$ is proportional to the displacement divergence via the fluid compressibility as :

$$
P=-\frac{1}{\chi} \frac{\partial u_{i}}{\partial x_{i}}
$$

In the proposed developments, we assume no relaxation phenomena (no specific time dependence) within the considered fluids. The stress then can be written considering those different elements, yielding the following expression :

$$
T_{i j}=-\left(P+j \omega\left(\frac{2}{3} \eta-\zeta\right) S_{k k}\right) \delta_{i j}+2 j \omega \eta S_{i j}
$$


where $T_{i j}$ and $S_{i j}$ respectively correspond to the stress and strain tensors, and $\omega$ is the angular frequency. One can remark that for $\eta=\zeta=0$ (no viscosity effects in the fluid), eq.(2) reduces to the classical pressure equilibrium with no shear effects $\left(T_{i j}=0\right.$ for $\left.i \neq j\right)$. According to the literature [4,5], the compressive viscosity may be responsible for important effects and hence should absolutely not be neglected. For instance, in the case of water, the absolute viscosity equals 0.8 centiPoise (cP) but the compressive viscosity is about 2.8 times larger [5]. These two parameters are then consider in the numerical applications of section III without any restriction and their respective influence on wave damping is evaluated.

\section{II.2 Fahmy-Adler formulation for viscous fluids}

It is now explain how one can represent the propagation of acoustic waves in viscous fluids as an eigenvector problem conformably to the general description of Fahmy-Adler [6] for solids. Without any loss of generality, one considers the propagation in the plane $\left(x_{1}, x_{3}\right)$ and the dependence along $x_{2}$ is given by the system to solve, as shown further. We assume an harmonic dependence along time, omitting to report the implicit term $e^{j \omega t}$. We define the following state vector, mixing displacement and stress components in the propagation plane :

$$
\mathbf{h}=\left\langle\begin{array}{llllll}
\frac{T_{21}}{j \omega} & \frac{T_{22}}{j \omega} & \frac{T_{23}}{j \omega} & u_{1} & u_{2} & u_{3}
\end{array}\right\rangle
$$

In a very general approach, one should add the electrical potential and the electrical displacement vector component along $x_{2}$, enabling one to represent dielectric viscous fluids. For the sake of simplicity, we only focus on the acoustic contribution. The propagation equations provide the first derivatives of the in-plane stresses versus $x_{2}$ as follows, using the notation $\sigma_{i j}=T_{i j} / j \omega$ :

$$
\frac{\partial}{\partial x_{2}}\left\{\begin{array}{l}
\sigma_{21} \\
\sigma_{22} \\
\sigma_{23}
\end{array}\right\}=j \rho \omega[I]\left\{\begin{array}{l}
u_{1} \\
u_{2} \\
u_{3}
\end{array}\right\}-\frac{\partial}{\partial x_{1}}\left\{\begin{array}{l}
\sigma_{11} \\
\sigma_{12} \\
\sigma_{13}
\end{array}\right\}-\frac{\partial}{\partial x_{3}}\left\{\begin{array}{l}
\sigma_{31} \\
\sigma_{32} \\
\sigma_{33}
\end{array}\right\}
$$

We now need three more equation to establish the first derivatives of the displacement versus $x_{2}$. This is performed by developing (2) as follows :

$$
\left\{\begin{array}{l}
\sigma_{11} \\
\sigma_{12} \\
\sigma_{13}
\end{array}\right\}=\frac{\partial}{\partial x_{1}}\left[A_{11}\right]\left[\begin{array}{l}
u_{1} \\
u_{2} \\
u_{3}
\end{array}\right\}+\frac{\partial}{\partial x_{2}}\left[A_{12}\right]\left\{\begin{array}{l}
u_{1} \\
u_{2} \\
u_{3}
\end{array}\right\}+\frac{\partial}{\partial x_{3}}\left[A_{13}\right]\left\{\begin{array}{l}
u_{1} \\
u_{2} \\
u_{3}
\end{array}\right\}
$$




$$
\begin{aligned}
& \left.\left\{\begin{array}{l}
\sigma_{21} \\
\sigma_{22} \\
\sigma_{23}
\end{array}\right\}=\frac{\partial}{\partial x_{1}}{ }^{t}\left[A_{12}\right]\left\{\begin{array}{l}
u_{1} \\
u_{2} \\
u_{3}
\end{array}\right\}+\frac{\partial}{\partial x_{2}}\left[A_{22}\right]\left\{\begin{array}{l}
u_{1} \\
u_{2} \\
u_{3}
\end{array}\right\}+\frac{\partial}{\partial x_{3}}\left[A_{23}\right]\right\}\left\{\begin{array}{l}
u_{1} \\
u_{2} \\
u_{3}
\end{array}\right\} \\
& \left\{\begin{array}{l}
\sigma_{31} \\
\sigma_{32} \\
\sigma_{33}
\end{array}\right\}=\frac{\partial}{\partial x_{1}}\left[A_{13}\right]\left\{\begin{array}{l}
u_{1} \\
u_{2} \\
u_{3}
\end{array}\right\}+\frac{\partial}{\partial x_{2}}\left[A_{23}\right]\left\{\begin{array}{l}
u_{1} \\
u_{2} \\
u_{3}
\end{array}\right\}+\frac{\partial}{\partial x_{3}}\left[A_{33}\right]\left\{\begin{array}{l}
u_{1} \\
u_{2} \\
u_{3}
\end{array}\right\}
\end{aligned}
$$

where the matrices $\left[A_{i j}\right]$ depends on the viscosity factors, the compressibility factor and the frequency (see Appendix A). From the $2^{\text {nd }}$ line of (5), one deduces the derivatives of the displacement versus $x_{2}$ :

$$
\frac{\partial}{\partial x_{2}}\left\{\begin{array}{l}
u_{1} \\
u_{2} \\
u_{3}
\end{array}\right\}=\left[A_{22}\right]^{-1}\left\{\left\{\begin{array}{l}
\sigma_{21} \\
\sigma_{22} \\
\sigma_{23}
\end{array}\right\}-\frac{\partial}{\partial x_{1}}\left[A_{12}\right]\left\{\begin{array}{l}
u_{1} \\
u_{2} \\
u_{3}
\end{array}\right\}-\frac{\partial}{\partial x_{3}}\left[A_{23}\right]\left[\begin{array}{l}
u_{1} \\
u_{2} \\
u_{3}
\end{array}\right\}\right\}
$$

Inserting (6) in (5) yields the following expressions of $\sigma_{1 j}$ et $\sigma_{3 j}$ :



$$
\begin{aligned}
& \left.\left.\left.\left\{\begin{array}{l}
\sigma_{31} \\
\sigma_{32} \\
\sigma_{33}
\end{array}\right\}=\frac{\partial}{\partial x_{1}}{ }^{t}\left[A_{13}\right]\left\{\begin{array}{l}
u_{1} \\
u_{2} \\
u_{3}
\end{array}\right\}+{ }^{t}\left[A_{23} \mathrm{I} A_{22}\right]^{-1}\left\{\left\{\begin{array}{l}
\sigma_{21} \\
\sigma_{22} \\
\sigma_{23}
\end{array}\right\}+\frac{\partial}{\partial x_{1}}{ }^{t}\left[A_{12}\right]\left\{\begin{array}{l}
u_{1} \\
u_{2} \\
u_{3}
\end{array}\right\}+\frac{\partial}{\partial x_{3}}\left[A_{23}\right]\right\}\left\{\begin{array}{l}
u_{1} \\
u_{2} \\
u_{3}
\end{array}\right\}\right\}+\frac{\partial}{\partial x_{3}}\left[A_{33}\right]\right\} \begin{array}{l}
u_{1} \\
u_{2} \\
u_{3}
\end{array}\right\}
\end{aligned}
$$

The above system is related to the state vector $h$ as follows :

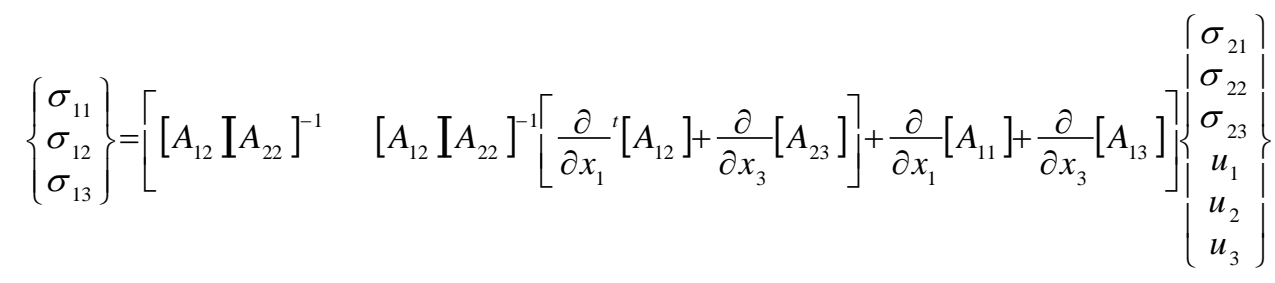

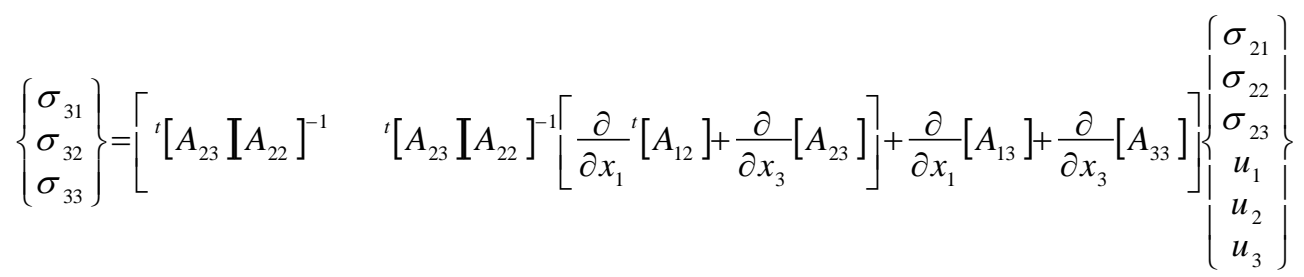

Inserting (8) in (4), and combining the result with (6), we obtain the eigenvalue formulation we are looking for. Assuming an harmonic dependence of the fields versus $x_{1}$ and $x_{3}$, replacing the corresponding gradients by $j \omega s_{1}$ and $j \omega s_{3}$ (with $s_{i}$ the slowness along $x_{i}$ ) yields : 




with

$$
\begin{gathered}
{\left[\alpha_{11}\right]=\left[\left(j \omega s_{1}\left[A_{12}\right]+j \omega s_{3}{ }^{t}\left[A_{23}\right]\right)\left[A_{22}\right]^{-1}\right]} \\
{\left[\alpha_{12}\right]=\left\{\begin{array}{c}
j \omega \rho[I]-j \omega s_{1}\left(\left[A_{12}\right]\left[A_{22}\right]^{-1}\left(j \omega s_{1}{ }^{t}\left[A_{12}\right]+j \omega s_{3}\left[A_{23}\right]\right)+j \omega\left(s_{1}\left[A_{11}\right]+s_{3}\left[A_{13}\right]\right)\right) \\
-j \omega s_{3}\left({ }^{t}\left[A_{23}\right]\left[A_{22}\right]^{-1}\left(j \omega s_{1}{ }^{t}\left[A_{12}\right]+j \omega s_{3}\left[A_{23}\right]\right)+j \omega\left(s_{1}\left[A_{13}\right]+s_{3}\left[A_{33}\right]\right)\right)
\end{array}\right]}
\end{gathered}
$$

The main difficulty introduced by the proposed development consists in the frequency dependence of the matrices $\left[A_{i j}\right]$, requiring their computation for each frequency point.

\section{II.3 Green's function of viscous fluids}

The way these relations can be used to compute the Green's function of the medium are detailed in $[7,8]$. Only a brief description of its derivation is reported here. Conformably to the Fahmy-Adler approach [6], one consider the above-defined state vector $\mathbf{h}$ to describe the acoustic properties of a semi-infinite or finite thickness medium potentially inserted in a stack of layer assuming flat interfaces. For a slowness set $\left(s_{1}, s_{3}\right)$, $\mathbf{h}$ can be represented as the product of matrix $\mathbf{F}$ composed of the 8 eigenvectors of the considered medium with a diagonal matrix $\Delta\left(x_{2}\right)$ of rank $8 \times 8$, which describes the dependence of the acoustic field versus $x_{2}$ via the eigenvalues $s_{2}$, and with a vector a corresponding to the amplitudes of the partial modes :

$$
\mathbf{h}\left(x_{2}\right)=\mathbf{F} \boldsymbol{\Delta}\left(x_{2}\right) \mathbf{a} \exp \left[j \omega\left(t-s_{1} x_{1}-s_{3} x_{3}\right)\right]
$$

For viscous fluids, the matrices $\mathbf{F}$ and $\mathbf{a}$ depend on frequency (contrarily to ideal fluids and classical solids). As in [7, 8,9], we introduce the variable $\mathbf{g}^{(m)}$ split in two parts to identify incident $\left(\mathbf{g}^{(\mathrm{m}-)}\right)$ and reflected $\left(\mathbf{g}^{(\mathrm{m}+)}\right)$ modes defined as follows :

$$
\mathbf{g}^{(m-)}\left(X_{m-1}\right)=\mathbf{R}^{(m)} \mathbf{g}^{(m+)}\left(X_{m-1}\right)
$$

where $m$ is the number of the considered layer in the stack ( $m=1$ for semi-infinite media) and $\mathbf{R}^{(\mathrm{m})}$ is a reflection matrix relating incident and reflected partial modes. The reflection 
matrices of each interface of the stack are then recursively deduced from the reflection matrix at the first interface $[8,9]$. This is achieved by introducing 2 sub-matrices $\mathbf{K}$ et $\mathbf{L}$ respectively associated to reflected and incident partial modes :

$$
\left[\mathbf{F}^{m+1}\right]^{-1} \mathbf{F}^{(m)}\left(\begin{array}{c}
I_{4} \\
\boldsymbol{\Delta}^{(m-)}\left(-t_{m}\right) \mathbf{R}^{(m)} \boldsymbol{\Delta}^{(m+)}\left(t_{m}\right)
\end{array}\right)=\left(\begin{array}{l}
\mathbf{K} \\
\mathbf{L}
\end{array}\right)
$$

with $t_{m}$ the thickness of the mth layer of the stack. These matrices then are used to compute the reflection matrix of the $(m+1)$ layer : $\mathbf{R}^{(m+1)}=\mathbf{L K}{ }^{-1}$. This recursive scheme is repeated until reaching the top layer of the stack (numbered $M$ ) at which we intend to compute the Green's function. The state vector $\mathbf{h}$ then reads :

$$
\begin{gathered}
\mathbf{h}\left(X_{M}\right)=\mathbf{F}^{(M)} \mathbf{g}^{(M)}\left(X_{M}\right)= \\
\mathbf{F}^{(M)}\left(\begin{array}{c}
I_{4} \\
\boldsymbol{\Delta}^{(M-)}\left(-t_{M}\right) \mathbf{R}^{(M)} \boldsymbol{\Delta}^{(M+)}\left(t_{M}\right)
\end{array}\right) \mathbf{g}^{(M+)}\left(X_{M}\right)=\left(\begin{array}{l}
\mathbf{N} \\
\mathbf{P}
\end{array}\right) \mathbf{g}^{(M+)}\left(X_{M}\right)
\end{gathered}
$$

In (13), we have defined two sub-matrices $\mathbf{N}$ et $\mathbf{P}$ respectively associated to the displacement field and to the stresses. The Green's function which relates $u_{i}$ to $T_{2 j}$ then is directly given by the product $\mathbf{G}=\mathbf{N P}^{-1}$. As in ref [10], we intend to compute the Green's function at the interface between two media. Since $\mathbf{G}$ can be computed following the above procedure at any surface or interface of a given material stack (still assuming flat parallel interfaces), it can be derived for any configurations (semi-infinite piezoelectric solids, plates, layered wave-guide in contact with viscous fluids in our case). In the case of a semi-infinite substrate in contact with a semi-infinite viscous fluid, $\mathbf{G}$ at the interface between the two media depends on frequency in a complicated way (due to viscosity effects). It is then computed for each frequency point as in the case of dispersive wave-guides. Note that in the spectral domain, the Green's function relates the generalized displacements $u_{i}[7,8,9]$ (including the potential $\phi$ as $u_{4}$ ) and the generalized surface stresses $T_{2 j}$ (where $T_{24}=D_{2}$ the electrical displacement normal to the surface) as $u_{i}=G_{i j} T_{2 j}$.

\section{II.4 Blötekjaër's harmonic admittance}

This Green's function can be used for computing the actual response of infinitely periodic guided elastic wave devices using different approaches $[9,10,11]$. In this work, we derive the celebrated effective permittivity [12] from the Green's function as follows : 


$$
\varepsilon_{E F F}\left(\omega, s_{1}\right)=\frac{1}{j|s| G_{44}\left(\omega, s_{1}\right)}
$$

yielding a frequency dependent effective permittivity. In (14), the expression of $\varepsilon_{E F F}$ is restricted to the saggital plane in which the waves is excited, assuming an infinite aperture of the transducer along $x_{3}$ ( $s_{3}$ then is set to zero). It is then used to compute an harmonic admittance [12], neglecting the mechanical contribution of the electrodes. This approach first used in [11] for interface wave computations enables one to simulate the excitation of acoustic waves by non-massive infinite periodic transducers (inter-digital transducers - IDT for instance) at any interface of any layered structure. In this section, we just point out the way the above effective permittivity is introduced in the calculation. The potential $\phi$ and electrical charge $Q$ are developed as Bloch-Floquet series to meet the periodic condition (see fig. 1 for axis definition) as follows :

$$
\begin{aligned}
\phi & =\sum_{n=-\infty}^{+\infty} \tilde{\phi}_{n} e^{-j \omega s_{n} x_{1}} \\
Q & =\sum_{n=-\infty}^{+\infty} \tilde{q}_{n} e^{-j \omega s_{n} x_{1}}
\end{aligned}
$$

where $s_{n}$ is the equivalent slowness of the nth term of the series, equal to $s_{1}+2 \pi n /(p \omega)$. The relation between the weights of $\phi$ and $Q$ is established in the spectral domain conformably to the effective permittivity definition [12] as follows :

$$
\tilde{q}_{n}=\omega\left|s_{n}\right| \varepsilon_{n} \tilde{\phi}_{n} \text { with } \varepsilon_{n}=\varepsilon_{E F F}\left(s_{n}\right)
$$

The boundary conditions of the addressed problem consists in the absence of charge between the electrodes and the nullity of the electrical field parallel to the excitation surface under the electrodes. The corresponding equations are solved thanks to a Legendre polynomial development of the unknown fields [12]. The weights of this development are related to the potential and charge coefficients as follows :

$$
\begin{gathered}
\omega s_{n} \tilde{\phi}_{n}=\sum_{m=M_{1}}^{M_{2}} \alpha_{m} \operatorname{sgn}(m-n) P_{n-m}\left(\cos \left(\frac{\pi a}{p}\right)\right) \\
\tilde{q}_{n}=\sum_{m=M_{1}}^{M_{2}} \beta_{m} P_{n-m}\left(\cos \left(\frac{\pi a}{p}\right)\right)
\end{gathered}
$$

where $P_{n}$ is the nth Legendre polynomial, $a$ is the width of the electrode and $p$ the grating period. The weights of the Legendre developments $\alpha_{m}$ and $\beta_{m}$ then becomes the actual unknown of the problem. $M_{1}$ and $M_{2}$ are the bounds of the discrete summation of (17), 
theoretically equal to - and + infinity. Practically, infinite sums cannot be handled for computation and the summation of (15) and (17) have to be truncated ( $N_{1}$ and $N_{2}$ represent the finite bounds of the sum in (15)). For a given value of $\omega$, the effective permittivity is known to tend toward an asymptotic value written $\varepsilon_{\propto}$. This is correct for usual materials, but a particular care must be attached to the case of viscous fluids, for which the frequency is intricately related to the intermediate matrix $\left[A_{i j}\right]$ used to derive the Green's function. However, for usual frequencies (smaller than $10 \mathrm{GHz}$ ), it is possible to find a slowness large enough to allow for a satisfying definition of $\varepsilon_{\infty}$ (i.e. for which the latter value of is moderately affected by the frequency). By properly choosing the values of $\left(M_{1}, M_{2}\right)$ and $\left(N_{1}\right.$, $N_{2}$ ) [12], one can establish the following relation between $\alpha_{m}$ and $\beta_{m}$ :

$$
\operatorname{sgn}(n) \beta_{m}=\varepsilon_{\infty} \operatorname{sgn}(n-m) \alpha_{m}
$$

Combining (17) and (18) yields the following homogeneous algebraic system which must be solved in our case for each frequency point of the development :

$$
\left[\left(\operatorname{sgn}(n-m)-\frac{\varepsilon_{\infty}}{\varepsilon_{n}} \operatorname{sgn}(n)\right) P_{n-m}\left(\cos \left(\frac{\pi a}{p}\right)\right)\right]\left\{\alpha_{m}\right\}=0
$$

Once the coefficients derived from (19), the computation of the harmonic potential $V_{\text {harm }}$ and current $I_{\text {harm }}$ is performed as described in [12], yielding the definition of the harmonic admittance as the ratio of $I_{\text {harm }}$ over $V_{\text {harm }}$. The computation of this value allows for the identification of coupled acoustic waves generated by non-massive IDTs and their characterization (phase velocity, coupling strength, propagation loss, reflection coefficient). In the next paragraph, we report numerous examples for which the influence of viscous fluids on different wave-guide is analysed.

\section{Computation results}

Calculations have been performed for different kind of wave polarisation on the most used piezoelectric substrates, i.e. quartz, lithium tantalate and lithium niobate, assuming a semiinfinite dielectric fluid domain (water) adjacent to the solid substrate. An isotropic dielectric constant equal to $10 \mathrm{pF} / \mathrm{m}$ was arbitrarily set for the fluid. This explains the change in static capacitance when passing from vacuum to the fluid load on the following admittance plots. 
The absolute viscosity of water (close to $1 \mathrm{cP}$ [8]) was changed from $1 \mathrm{cP}$. to $10 \mathrm{cP}$. and 100 $\mathrm{cP}$. to check its influence on the wave characteristics. For all the tested materials, Rayleigh as well as leaky SAW have been considered. For quartz, surface transverse waves also have been taken into account. Finally, the influence of water load on acoustic plate modes (APM) on $(\mathrm{YXl}) / 36^{\circ}$ cut of quartz has been simulated for both viscous and non viscous water. Note that all the admittance results are given for a unit width aperture.

Figure 1 shows the general geometry of the problem. For the sake of consistence, a metal ratio equal to 0.5 was considered for all computations, and the period fixed to $5 \mu \mathrm{m}$ (acoustic wavelength forced to $10 \mu \mathrm{m})$. Figure 2 and 3 show the results obtained for the (YXl)/36 ${ }^{\circ}$ cut of quartz, expended in 3 sections to magnify the different contributions to the harmonic admittance.

The damping of Rayleigh wave by water load is clearly shown in fig.2. Also an influence is pointed out on the surface skimming bulk wave (SSBW) close to the Rayleigh wave and radiated from the surface, mainly due to the damped Rayleigh wave conductance. The very small influence of viscosity on the Rayleigh wave signature yields almost no difference between viscous and non viscous water loaded admittance.

Figure $3(a \& b)$ show the influence of water load on the two leaky modes (fast shear and longitudinal) on (YXl) $/ 36^{\circ}$ cut of quartz, which is clearly less dramatic than in the case of the Rayleigh wave. These two modes partially guided by the surface exhibit almost pure polarisation and then a negligible component normal to the surface. This means that they radiate almost no energy in the fluid. However, they are affected by viscosity effects, yielding a significant increase of losses due to viscous shearing within the fluid. This is particularly emphasized for the fast shear leaky wave which is almost not affected by the presence of ideal water. In contact with viscous water, the conductance of this mode is reduced by more than a factor of two and the quality factor of the mode changes from 8300 to 3200 (this value is derived from the conductance peak relative to the mode as the ratio of its central frequency divided by its width at half height). In that particular case, the impact of rather small viscosity properties at usual frequencies cannot be neglected. One can note that the central frequency also is slightly shifted down. A more surprising result is the robustness of the longitudinal mode in contact with water. In that case, the mode guiding even seems improved according to the slight increase of the corresponding conductance peak. However, viscosity effects also 
dramatically reduce its excitation efficiency. Again in that case, they induce a slight frequency decrease of the mode. The values of the quality factor for the different analysed working conditions are respectively 2650 (vacuum), 3000 (ideal water) and 1850 (viscous water).

Figure 4 shows the same computations performed for STW on the (YXlt)/36 $/ 90^{\circ}$ quartz cut. The curves relative to vacuum and to non viscous water as adjacent media are superimposed, since no shear displacement can develop in perfect fluids. As soon as viscosity is introduced, the STW is dramatically damped, yielding a quality factor of 12400 which rapidly falls down when increasing the viscosity, as shown in fig.5. A quality factor of 2000 is predicted for a viscosity equal to $10 \mathrm{cP}$ and it passes under 500 when increasing the viscosity to $100 \mathrm{cP}$. This is an important issue to consider when expecting using the STW for immersed biomedical purpose, since liquids used for such application may exhibit rather large viscosity values.

The case of Love waves is also investigated to check whether the elimination of propagation loss associated to the STW allows for improving the device operation. In that purpose, we assume a fused silica overlay atop the quartz substrate to guide the pure shear wave. The electric excitation is assumed at the interface between the quartz substrate and the silica overlay. The thickness of the $\mathrm{SiO}_{2}$ layer was arbitrarily set to $500 \mathrm{~nm}$, yielding a Love wave propagating with a phase velocity close to $5010 \mathrm{~m}$.s-1 with a coupling coefficient (the usual $K_{s}^{2}$ [1]) equal to $2.1 \%$. As previously, a water load does not significantly change the harmonic admittance of the corresponding structure. However, viscosity effects again yield a dramatic reduction of the wave conductance, which then presents a finite quality factor. Note that for a $0.1 \mathrm{cP}$ viscosity, this factor equals 2100 but it falls down to 660 for $1 \mathrm{cP}$ (standard water). We also point out a frequency shift of about $250 \mathrm{kHz}$ between these two operating conditions. Love waves on quartz consequently are very sensitive to viscosity and seem hardly capable to operate with highly viscous fluids (viscosity larger than $50 \mathrm{cP}$ ) as loading medium.

Regarding these results, it then sounds interesting to analyse the robustness of longitudinally polarized waves in presence of fluids exhibiting such viscosities. In that matter, we have considered the case of (YZ) cut of lithium niobate, on which a well coupled Rayleigh wave can propagate but also a high velocity longitudinal wave may be trapped under thick electrodes. Even if we cannot simulate such an electrode configuration, we can point out the longitudinal wave signature on the harmonic admittance and then predict the influence of 
viscous fluids on this kind of wave. Figure 7 shows the influence of ideal and viscous water loads on the harmonic admittance signatures of the Rayleigh and longitudinal waves on (YZ) $\mathrm{LiNbO}_{3}$ cut. We also have reported the evolution of these responses for various values of viscosity in fig. 8 , which shows that the longitudinal wave is clearly less affected by viscosity than shear waves on quartz are. One could also argue that the Rayleigh wave signature dose not change when increasing the viscosity, but it should be kept in mind that this wave almost vanishes when loaded by water, whereas the amplitude of the longitudinal wave almost remain unchanged for the different considered operating conditions.

However, such longitudinal wave cannot be considered as a relevant demonstration of their interest for immersed applications since its signature on the harmonic admittance remains weak compared to a real surface-guided mode. We then investigate the operation of Lamb waves loaded by fluids. This is a very interesting configuration in which very thin plates simultaneously can support waves not damped by the water load because of their very small phase velocity (lower than $1500 \mathrm{~m} \cdot \mathrm{s}^{-1}$ ) and also high velocity waves that almost behave like pure longitudinal modes. Figure 9 shows the dispersion curves of Lamb waves on a (YZ) $\mathrm{LiNbO}_{3}$ cut plate. For thickness-frequency products smaller than $220 \mathrm{~m} . \mathrm{s}^{-1}$, the first antisymmetric mode A0 exhibits a phase velocity smaller than the one of water and also one can remark that the first symmetric mode (S0) velocity is almost constant and close to the longitudinal bulk wave velocity (about $7000 \mathrm{~m} \cdot \mathrm{s}^{-1}$ ). Note that for the considered wavelength, the polarisation of this mode principally lie along the $x$ axis (the elliptic polarisation actually is very weak in that case).

We check the behaviour of such a device for two plate thickness, i.e. 1.4 and $5 \mu \mathrm{m}$. We then report the previous dispersion curve considering these fixed thickness in fig.10 (a\&b). Since the wavelength is the inverse of the wave number plotted in fig.10, we directly deduce the operating frequencies of the different mode corresponding to a wave number equal to $100 \mathrm{~km}^{-}$ ${ }^{1}$. In the first case, the A0 mode is close to the velocity threshold at which the mode radiates its energy in water. However, due to the water mass loading, its contribution to the harmonic admittance is shifted down, yielding no leakage due to radiation in water (see fig.11(a)). Its electromechanical coupling is very weak but one should note that such a structure (a single crystal plate, symmetric around its neutral line) is poorly adapted to excite anti-symmetric waves. On the other hand, a coupling factor of more than $4 \%$ is obtained for the S0 mode. 
Figure 11(a) shows the degradation of the A0 mode amplitude when increasing the viscosity. An identical behaviour is pointed out for the S0 mode which is damped by water but still exhibit a well defined response (fig.11(b)). The mode amplitude dramatically falls down for values of viscosity larger or equal to $10 \mathrm{cP}$, but the A0 mode actually exhibits a sharper peak than the S0 does under these operating conditions. In that case, one can point out that the main issue is to efficiently excite the A0 mode for any exploitation of its properties. A bimorph structure (naturally non symmetric) would certainly help in that purpose.

For the $5 \mu \mathrm{m}$ thick plate, the situation is quite different, since the A0 mode is largely above the radiation threshold, and the $\mathrm{A} 1$ and $\mathrm{S} 1$ modes do contribute now to the harmonic admittance (see fig.10(b)). The same computations as previously performed are then conducted for that case. The A0 and S0 modes almost vanishes as soon as the plate is loaded by water (fig.12(a)). However, the A1 mode exhibit more robustness to the presence of water, as shown in fig.12(b). It still exhibits a well defined peak on the conductance even for $10 \mathrm{cP}$ viscosity. This result tends to prove that high order Lamb modes could operate as well as a low velocity A0 mode even when submitted to water load. This present the advantage of an efficient excitation together with a device architecture simpler than the one required for the exploitation of the A0 mode (very thin bimorph structures).

\section{Conclusion}

A model describing the propagation of acoustic waves in fluids exhibiting or not viscosity properties has been developed and implemented. The use of an harmonic admittance enables one to determine the influence of non viscous and viscous water load on various combination of waves/substrates. The damping of Rayleigh wave due to the radiation of the displacement field component normal to the guiding surface is clearly pointed out. It is also shown that waves exhibiting a quasi pure shear polarisation are almost not affected by the presence of water. However, the influence of viscosity appears even for pure shear waves on quartz, yielding an additional leakage phenomenon generally neglected for practical applications. A more unusual result is the robustness of longitudinally polarized waves versus the presence of water. Moreover, according to our computations, since no shear effect is involved in this kind of propagation, longitudinal modes or pseudo-modes would be less sensitive to viscosity than the shear waves are. Also Lamb waves were tested, showing the interest of the A0 mode on 
very thin plates but also the capability of higher order modes to operate even with moderately viscous water loads.

These results suggest that contrarily to what is usually admitted (i.e., only pure shear wave and modes with a velocity smaller than the water threshold $-1500 \mathrm{~m} \cdot \mathrm{s}^{-1}$ ), different kind of waves and modes can be exploited for immersed application even with moderately viscous fluids. This mean there is still a lot of opportunities to point out optimal combinations of material, wave nature and electrode structures for the development of electro-acoustic devices operating in contact with fluids (for instance, sensing applications within organic bodies).

In this context, the simulation of more complicated electrode structures using a combination of finite element analysis boundary element methods would yield better insight an new ideas in the development of such devices.

Acknowledgements : Special thanks are due to J.B. Briot, Th. Pastureaud, R. Lardat and W. Steichen for fruitful discussions. 


\section{Appendix A : Definition of the matrices $\left[A_{i j}\right]$}

We express the stress field according to (2) to define the matrices that appear in (5) as follows:

$$
\begin{aligned}
& \left\{\begin{array}{l}
T_{11} \\
T_{12} \\
T_{13}
\end{array}\right\}=\frac{\partial}{\partial x_{1}}\left\{\begin{array}{ccc}
\frac{1}{\chi}+j\left(\frac{4}{3} \eta+\zeta\right) \omega & 0 & 0 \\
0 & j \omega \eta & 0 \\
0 & 0 & j \omega \eta
\end{array}\right\}\left\{\begin{array}{l}
u_{1} \\
u_{2} \\
u_{3}
\end{array}\right\}+ \\
& \frac{\partial}{\partial x_{2}}\left[\begin{array}{ccc}
0 & \frac{1}{\chi}-j\left(\frac{2}{3} \eta-\zeta\right) \omega & 0 \\
j \omega \eta & 0 & 0 \\
0 & 0 & 0
\end{array}\right]\left\{\begin{array}{l}
u_{1} \\
u_{2} \\
u_{3}
\end{array}\right\}+\frac{\partial}{\partial x_{3}}\left[\begin{array}{ccc}
0 & 0 & \frac{1}{\chi}-j\left(\frac{2}{3} \eta-\zeta\right) \\
0 & 0 & 0 \\
j \omega \eta & 0 & 0
\end{array}\right\} \mid\left\{\begin{array}{l}
u_{1} \\
u_{2} \\
u_{3}
\end{array}\right\}
\end{aligned}
$$

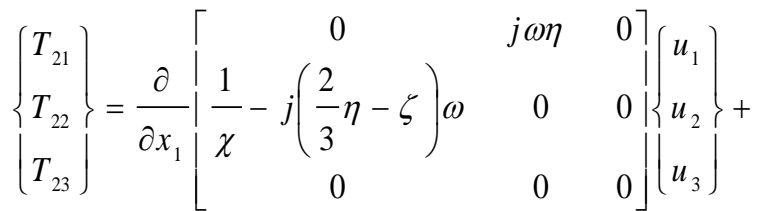

$$
\begin{aligned}
& \left.\frac{\partial}{\partial x_{2}}\left[\begin{array}{ccc}
j \omega \eta & 0 & 0 \\
0 & \frac{1}{\chi}+j\left(\frac{4}{3} \eta+\zeta\right) \omega & 0 \\
0 & 0 & j \omega \eta
\end{array}\right]\left\{\begin{array}{l}
u_{1} \\
u_{2} \\
u_{3}
\end{array}\right\}+\frac{\partial}{\partial x_{3}}\left[\begin{array}{ccc}
0 & 0 & 0 \\
0 & 0 & \frac{1}{\chi}-j\left(\frac{2}{3} \eta-\zeta\right) \\
0 & j \omega \eta & 0
\end{array}\right] \mid \begin{array}{l}
u_{1} \\
u_{2} \\
u_{3}
\end{array}\right\} \\
& \left.\left\{\begin{array}{l}
T_{31} \\
T_{32} \\
T_{33}
\end{array}\right\}=\frac{\partial}{\partial x_{1}}\left\{\begin{array}{ccc}
0 & 0 & j \omega \eta \\
\frac{1}{\chi}-j\left(\frac{2}{3} \eta-\zeta\right) \omega & 0 & 0
\end{array}\right] \mid \begin{array}{l}
u_{1} \\
u_{2} \\
u_{3}
\end{array}\right\}+ \\
& \frac{\partial}{\partial x_{2}}\left[\begin{array}{ccc}
0 & 0 & 0 \\
0 & 0 & j \omega \eta \\
0 & \frac{1}{\chi}-j\left(\frac{2}{3} \eta-\zeta\right) \omega & \mathbf{0}
\end{array}\right]\left\{\begin{array}{l}
u_{1} \\
u_{2}
\end{array}\right\}+\frac{\partial}{\partial x_{3}}\left[\begin{array}{cccc}
j \omega \eta & 0 & 0 \\
0 & j \omega \eta & 0 \\
0 & 0 & \frac{1}{\chi}+j\left(\frac{4}{3} \eta+\zeta\right)
\end{array}\right] \omega \mid\left\{\begin{array}{l}
u_{1} \\
u_{2} \\
u_{3}
\end{array}\right\}
\end{aligned}
$$

Consequently, 6 independent matrices are defined allowing for a more compact matrix formulation of the problem : 


$$
\begin{aligned}
& {\left[A_{11}\right]=\left[\begin{array}{ccc}
\frac{1}{j \chi \omega}+\frac{4}{3} \eta & 0 & 0 \\
0 & \eta & 0 \\
0 & 0 & \eta
\end{array}\right]\left[A_{12}\right]=\left[\begin{array}{ccc}
0 & \frac{1}{j \chi \omega}-\frac{2}{3} \eta & 0 \\
\eta & 0 & 0 \\
0 & 0 & 0
\end{array}\right]\left[A_{13}\right]=\left[\begin{array}{ccc}
0 & 0 & \frac{1}{j \chi \omega}-\frac{2}{3} \eta \\
0 & 0 & 0 \\
\eta & 0 & 0
\end{array}\right]} \\
& {\left[A_{22}\right]=\left[\begin{array}{ccr}
\eta & 0 & 0 \\
0 & \frac{1}{j \chi \omega}+\frac{4}{3} \eta & 0 \\
0 & 0 & \eta
\end{array}\right]\left[A_{23}\right]=\left[\begin{array}{ccc}
0 & 0 & 0 \\
0 & 0 & \frac{1}{j \omega \chi}-\frac{2}{3} \eta \\
0 & \eta & 0
\end{array}\right]\left[A_{33}\right]=\left[\begin{array}{ccc}
\eta & 0 & 0 \\
0 & \eta & 0 \\
0 & 0 & \frac{1}{j \chi \omega}+\frac{4}{3} \eta
\end{array}\right]}
\end{aligned}
$$

$$
\left[A_{21}\right]^{\prime}\left[A_{12}\right]\left[A_{31}\right]^{\prime}\left[A_{13}\right]\left[A_{32}\right\}^{t}\left[A_{23}\right]
$$




\section{References :}

[1] D. Royer, E. Dieulesaint, "Elastic waves in solids", Springer-Verlag Ed., 2000

[2] J. Kestin, R. Dipippo, "Chapter 2r. :Viscosity of Gases" in "American Institute of Physics Handbook”, 3rd Ed., McGraw-Hill, 1982

[3] R.N. Thurston, "Wave propagation in fluids and solids", in Physical Acoustics, Vol. 1A, W.P. Mason Ed., Academic Press, 1964

[4] W.P. Mason, "Piezoelectric crystals and mechanical resonators", in Physical Acoustics, Vol. 1A, W.P. Mason Ed., Academic Press, 1964

[5] T.A. Litovitz, C.M. Davis, "Structural and shear relaxation in liquids" in Physical Acoustics, Vol. 2A, W.P. Mason Ed., Academic Press, 1965

[6] A. H. Fahmy and E. Adler, "Propagation of surface acoustic waves in multilayer: a matrix description", Appl. Phys. Lett., 22, 495-497 (1973)

[7] Th. Pastureaud, V. Laude, S. Ballandras, "Stable scaterring matrix method for surface acoustic waves in piezoelectric multilayers”, Appl. Phys. Lett., Vol. 80, pp. 2544-2546, 2002

[8] A. Reinhardt, Th Pastureaud, V. Laude, S. Ballandras, "Scattering matrix method for acoustic waves in piezoelectric, fluid, and metallic multilayers", J. Appl. Phys., Vol. 94, pp. 6923-6931, 2003

[9] S.Ballandras, A. Reinhardt, V. Laude, A. Soufyane, S. Camou, W. Daniau, T. Pastureaud, W. Steichen, R. Lardat, M. Solal, P. Ventura, "Simulations of surface acoustic wave devices built on stratified media using a mixed finite element/boundary integral formulation”, J. Appl. Phys., Vol. 96, n¹2, pp. 7731-7741, 2004

[10] S. Camou, V. Laude, Th. Pastureaud, S. Ballandras, "Interface acoustic wave properties in some common crystal cuts", IEEE Trans. on UFFC, Vol. 50, n¹0, pp. 1363-1370, 2003.

[11] G. Endoh, K. Hashimoto, M. Yamaguchi, "SAW propagation characterization by finite element method and spectral domain analysis", Jpn. J. Appl. Phys., 34, 5B, pp. 2638 $2641(1995)$.

[12]K. Blötekjaer, K. A. Ingebrigsten, and H. Skeie, "A method for Analysing Waves in Structures Consisting of metallic Strips on Dispersive Media”, IEEE Trans. on Electron. Devices, Vol. ED-20, pp. 1133-1138 (1973). 
Figure captions :

Fig.1 Scheme of the typical geometry considered for simulations

Fig.2 Harmonic admittance of an infinite periodic IDT on (YXl) $/ 36^{\circ}$ cut of quartz with an adjacent semi-infinite fluid domain, influence on the Rayleigh and surface skimming bulk wave (SSBW) signature (period $5 \mu \mathrm{m}$, metallisation ratio $a / p=0,5$ )

Fig.3 Harmonic admittance of an infinite periodic IDT on $(\mathrm{YXl}) / 36^{\circ}$ cut of quartz with vacuum, ideal and viscous water as adjacent semi-infinite media, (a) shear and (b) longitudinal radiated bulk waves (period $5 \mu \mathrm{m}$, metallisation ratio $a / p=0,5$ )

Fig.4 Harmonic admittance of an infinite periodic IDT on (YXlt) $/ 36^{\circ} / 90^{\circ}$ cut of quartz (STW cut) with vacuum, ideal and viscous water as adjacent semi-infinite media (period $5 \mu \mathrm{m}$, metallisation ratio $a / p=0,5$ )

Fig.5 Influence of the viscosity on the STW response on (YXlt) $/ 36^{\circ} / 90^{\circ}$ quartz - absolute viscosity equal to $1 \mathrm{cP}, 10 \mathrm{cP}$ and $100 \mathrm{cP}$ (compressive viscosity equals 2.8 times the absolute one)

Fig.6 Harmonic admittance of an infinite periodic IDT at the interface between a 500nm thick $\mathrm{SiO}_{2}$ overlay and the (YXlt)/36\% $/ 90^{\circ}$ cut of quartz (excitation of Love waves) with vacuum, ideal and viscous water $(0.1$ and $1 \mathrm{cP})$ as adjacent semi-infinite media (period $5 \mu \mathrm{m}$, metallisation ratio $a / p=0,5,500 \mathrm{~nm}$ thick fused quartz overlay)

Fig.7 Harmonic admittance of an infinite periodic IDT on (YZ) cut of $\mathrm{LiNbO}_{3}$ with vacuum, ideal and viscous water as adjacent semi-infinite media (period $5 \mu \mathrm{m}$, metallisation ratio $a / p=0,5)$

Fig. 8 Influence of the viscosity on the Rayleigh wave and longitudinal wave responses on (YZ) $\mathrm{LiNbO}_{3}$ - absolute viscosity equal to $1 \mathrm{cP}, 10 \mathrm{cP}$ and $100 \mathrm{cP}$ (compressive viscosity equals 2.8 times the absolute one)

Fig.9 Dispersion curves of lamb waves on a (YZ) lithium niobate thin plate 
Fig.10 Dispersion curves of fig.9 for a plate thickness fixed to (a) $1.4 \mu \mathrm{m}$ (b) $5 \mu \mathrm{m}$

Fig.11 Harmonic admittance of an infinite periodic IDT on a $1.4 \mu \mathrm{m}$ thick plate of $\mathrm{LiNbO}_{3}$ (YZ) cut with vacuum, ideal and viscous water $(1 \mathrm{cP}$ and $10 \mathrm{cP}$, compressive viscosity equals 2.8 times the absolute one) as adjacent semi-infinite media, (a) A0 mode (b) S0 mode (period $5 \mu \mathrm{m}$, metallisation ratio $a / p=0,5$ )

Fig.12 Harmonic admittance of an infinite periodic IDT on a $5 \mu \mathrm{m}$ thick plate of $\mathrm{LiNbO}_{3}(\mathrm{YZ})$ cut with vacuum, ideal and viscous water $(1 \mathrm{cP}$ and $10 \mathrm{cP}$, compressive viscosity equals 2.8 times the absolute one) as adjacent semi-infinite media, (a) A0 and S0 modes (b) A1 and S1 modes (period $5 \mu \mathrm{m}$, metallisation ratio $a / p=0,5$ ) 


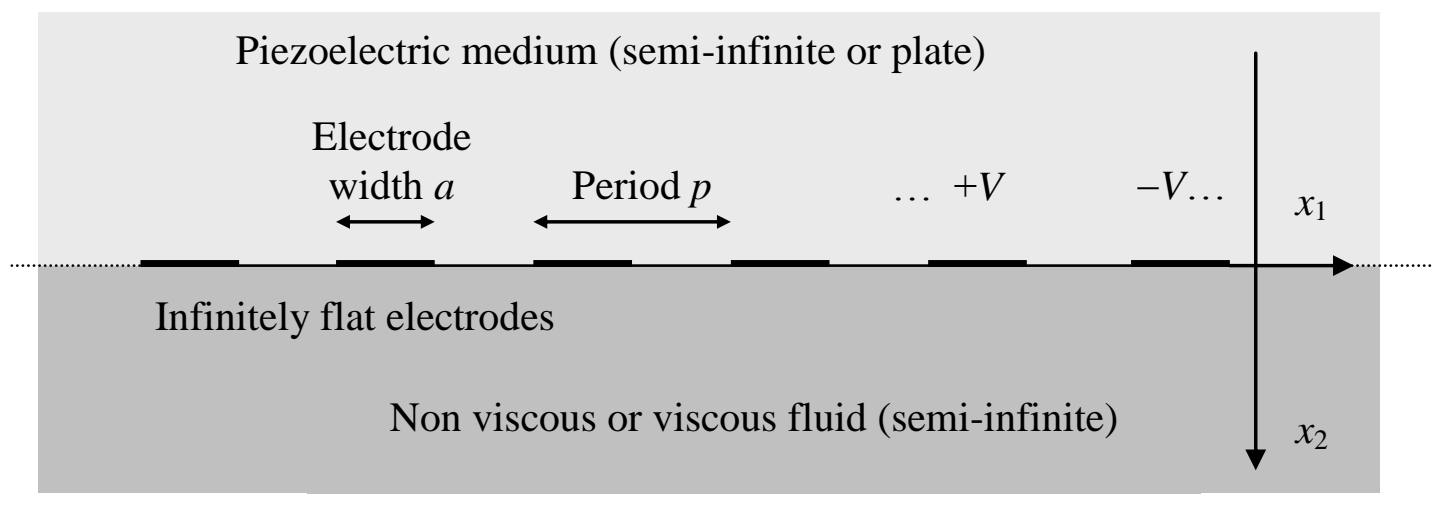

Fig.1 


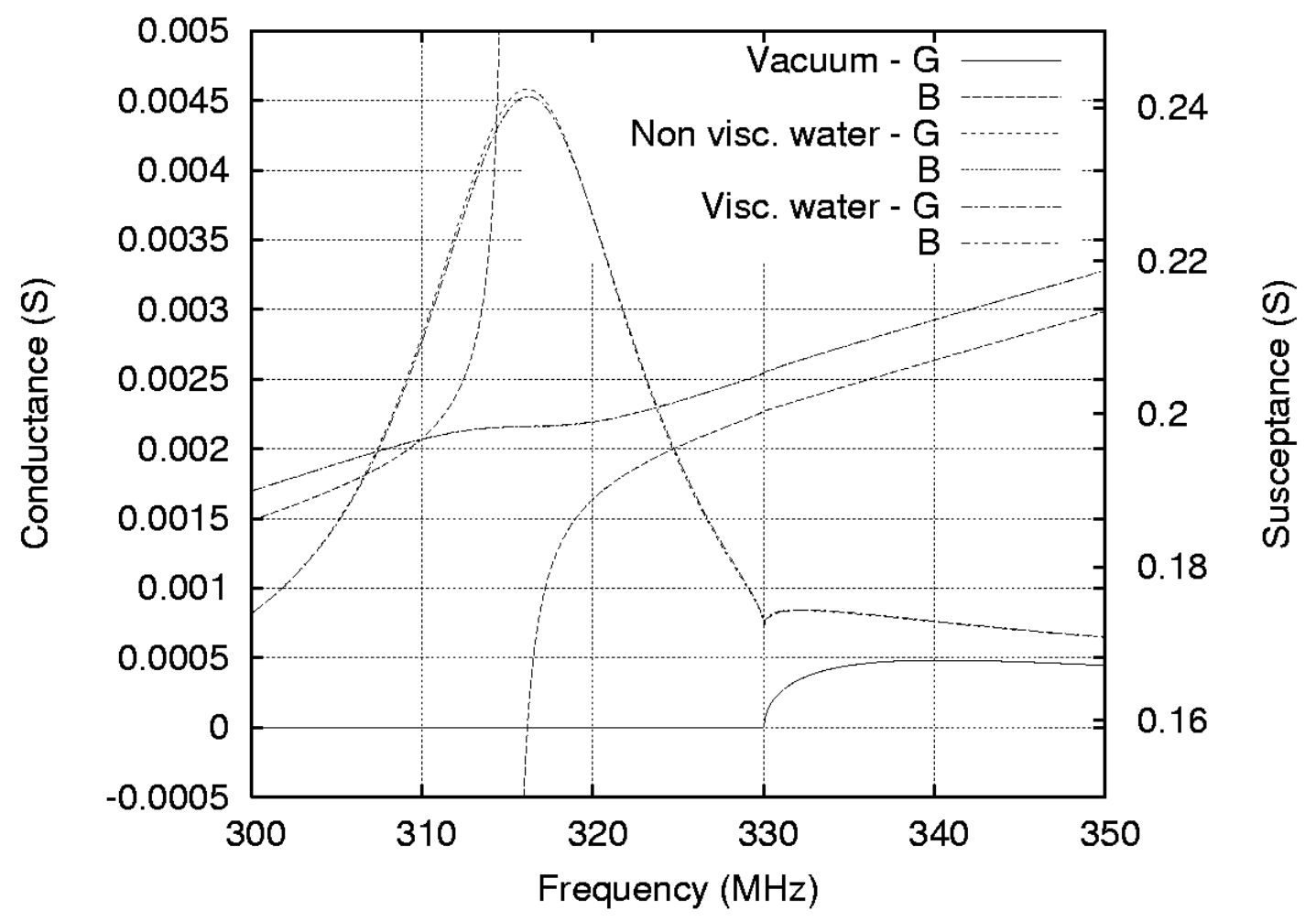

Fig.2 


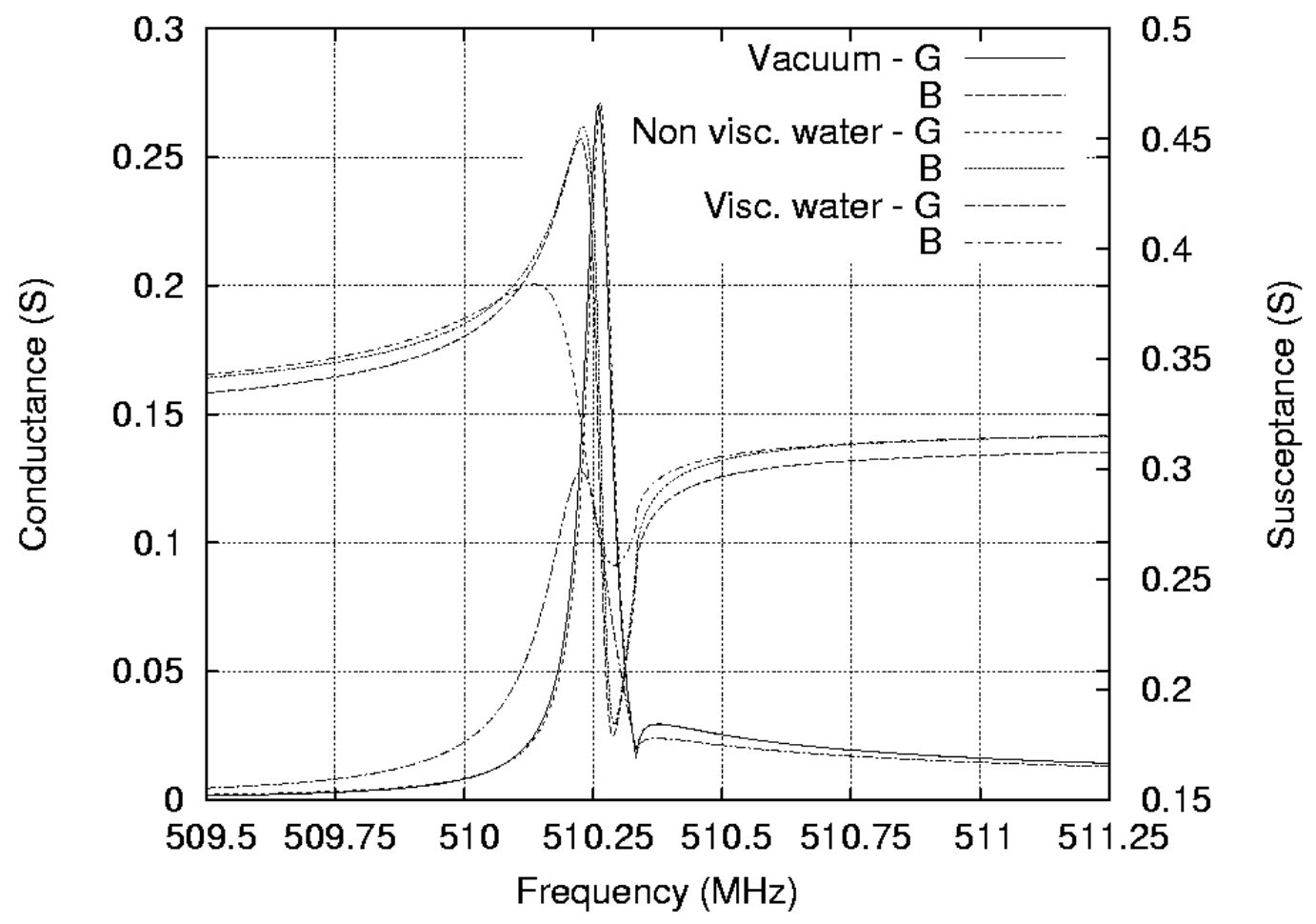

(a)

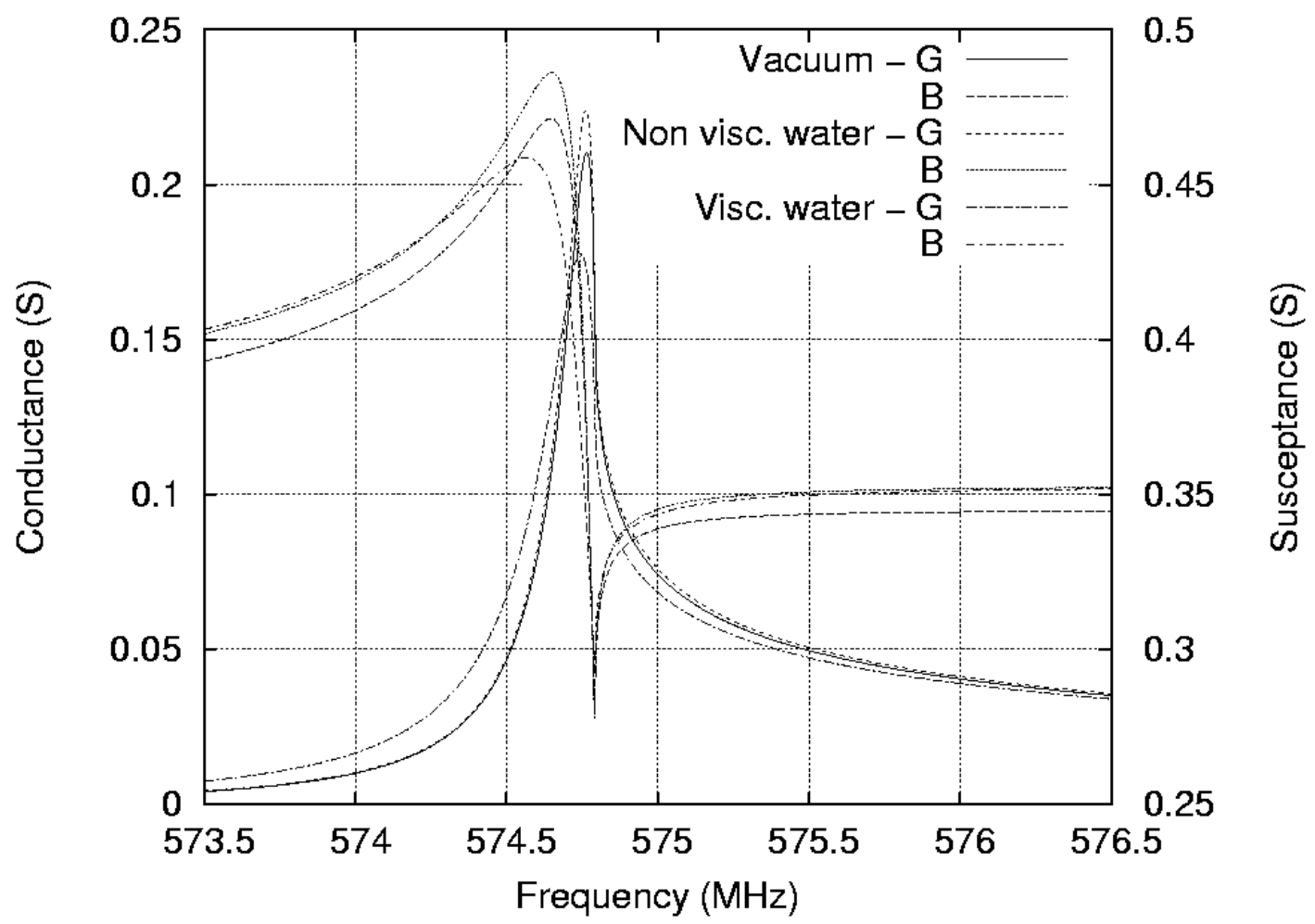

(b)

Fig.3 


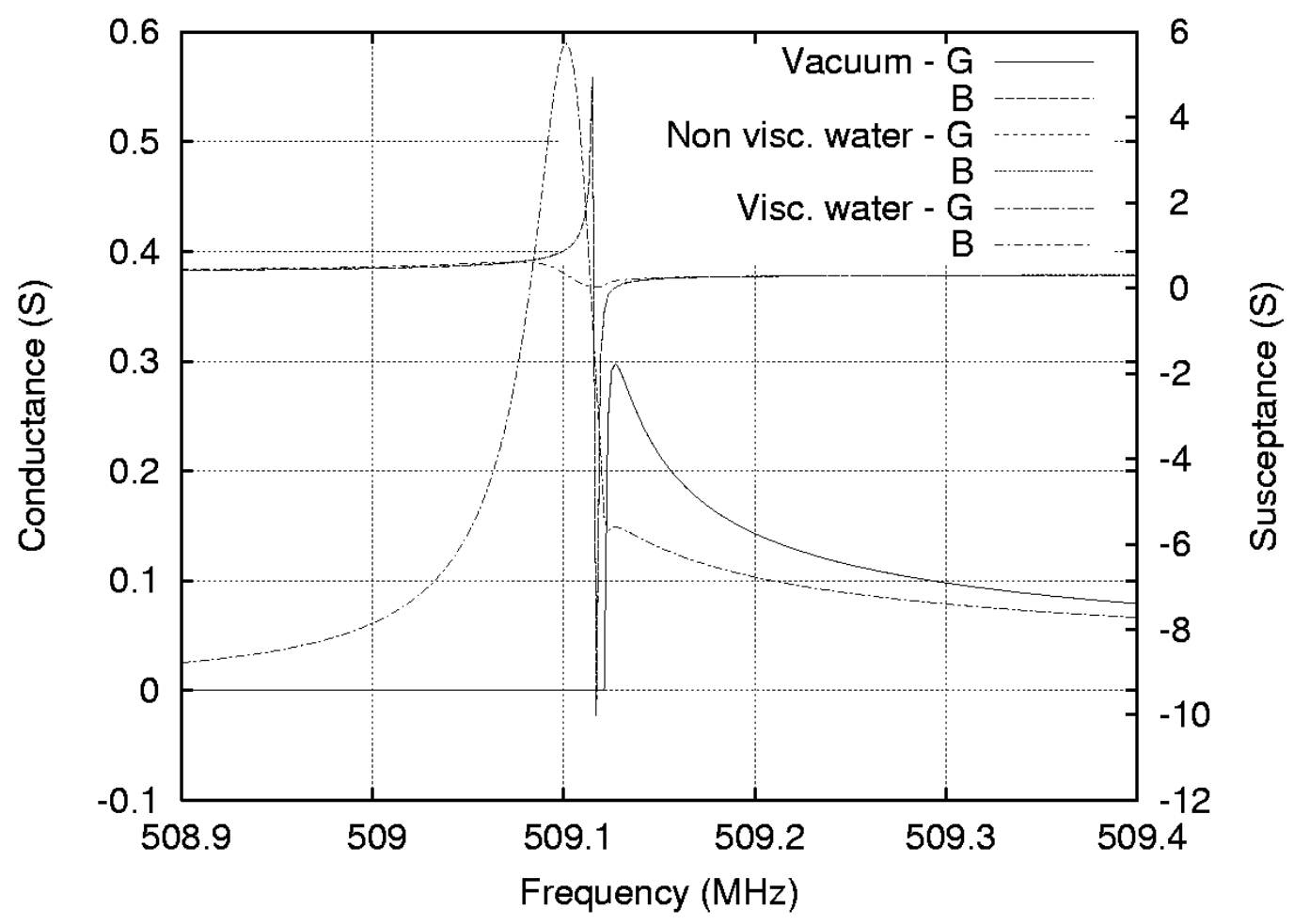

Fig.4

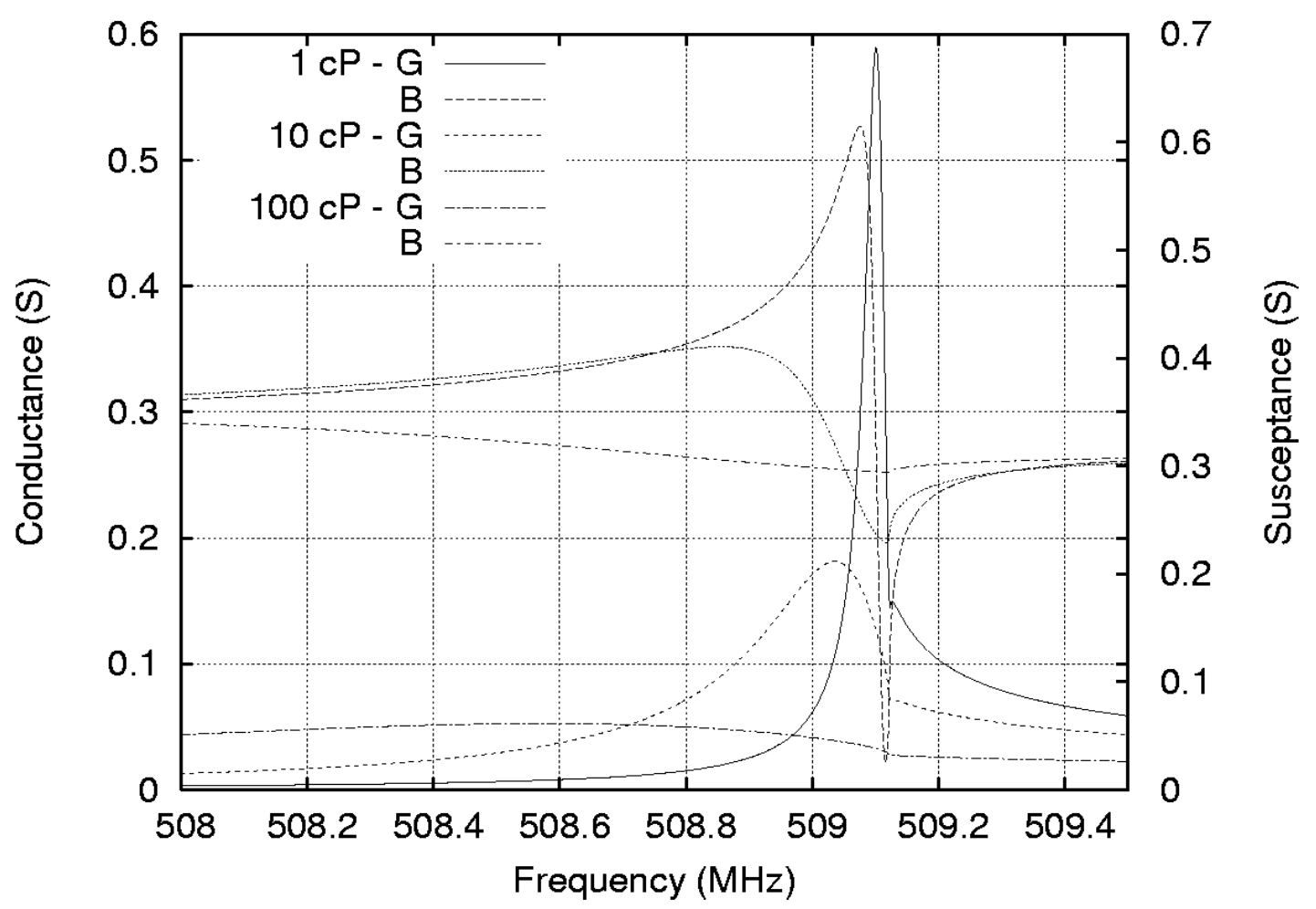

Fig.5 


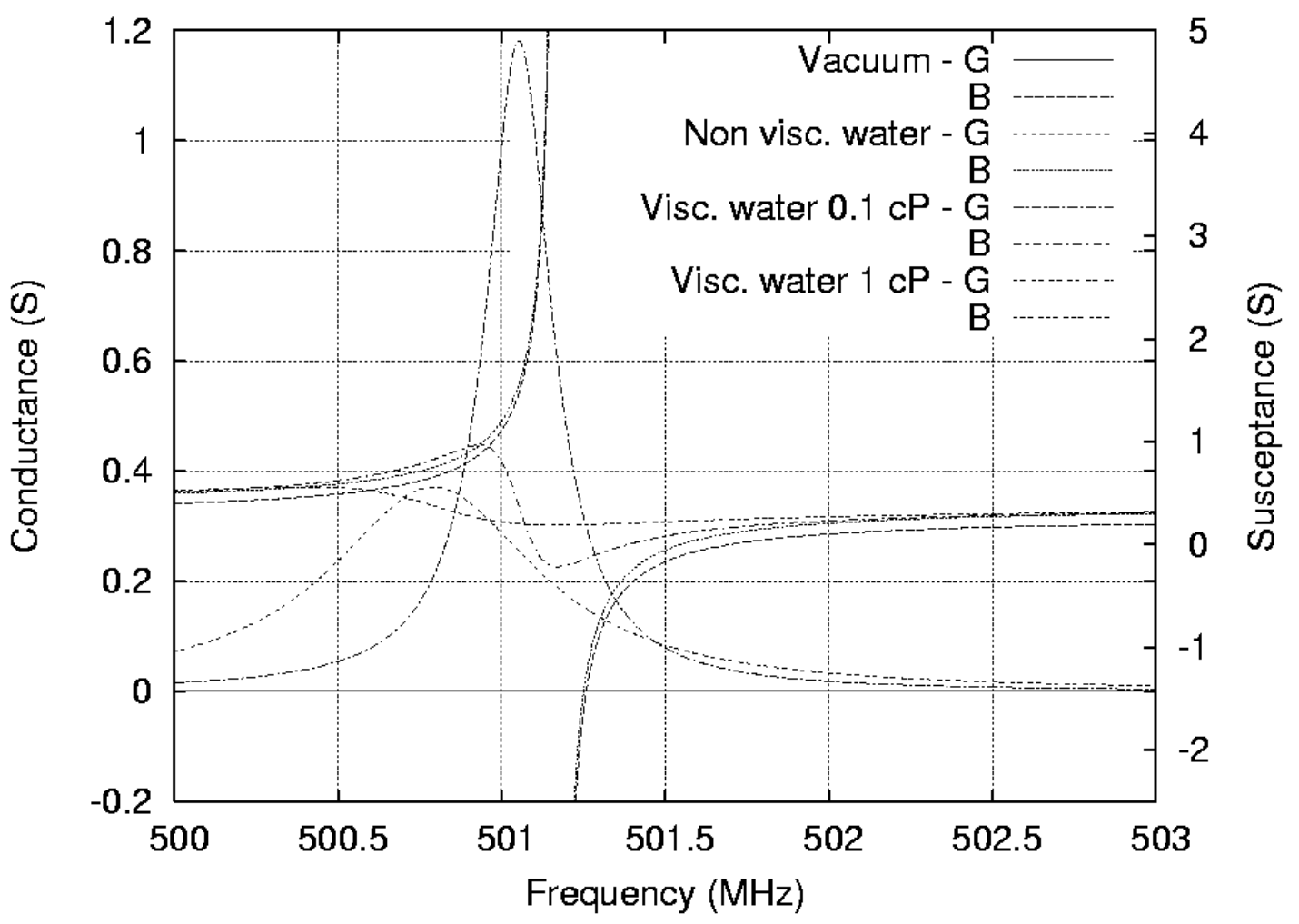

Fig.6 


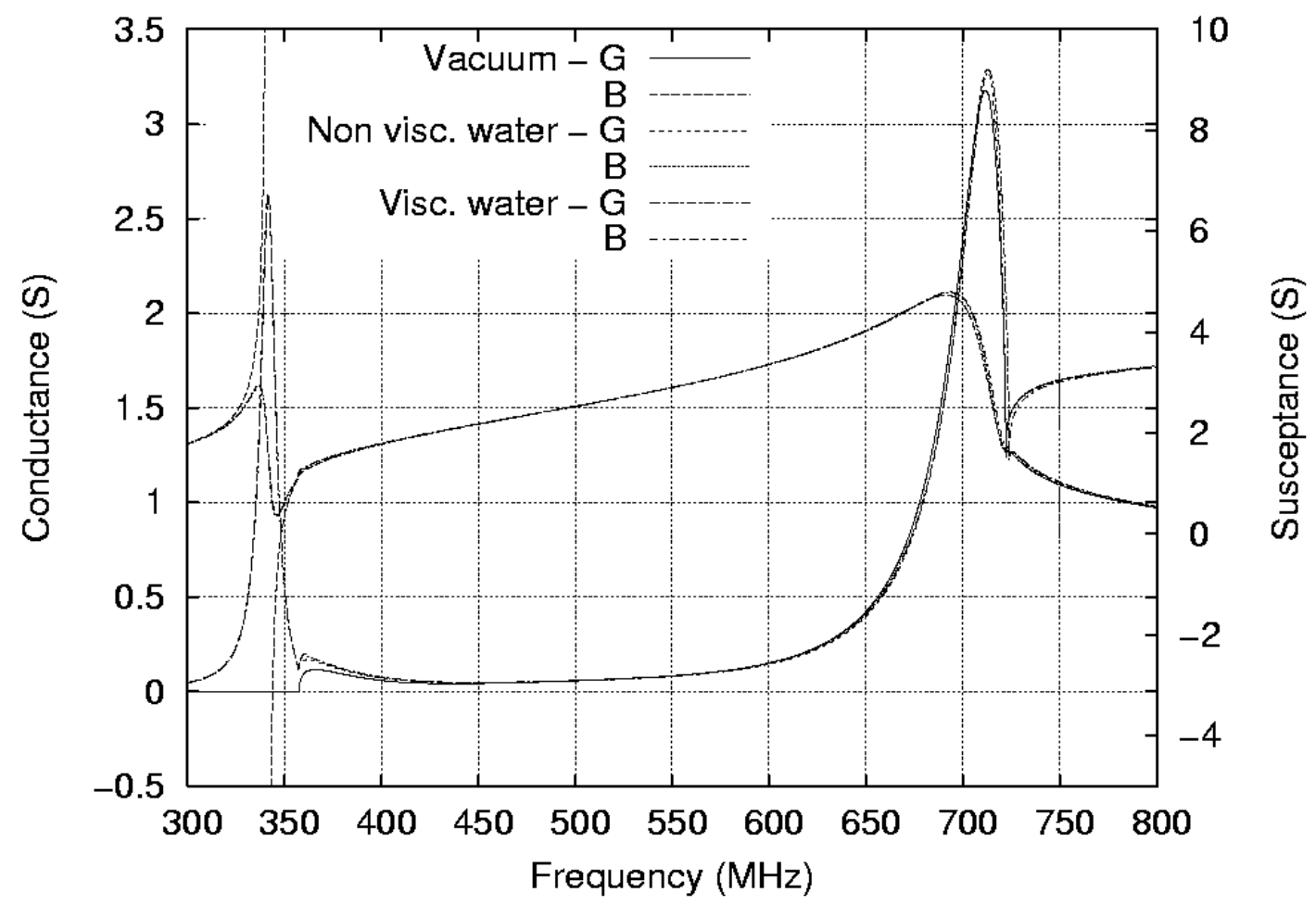

Fig.7

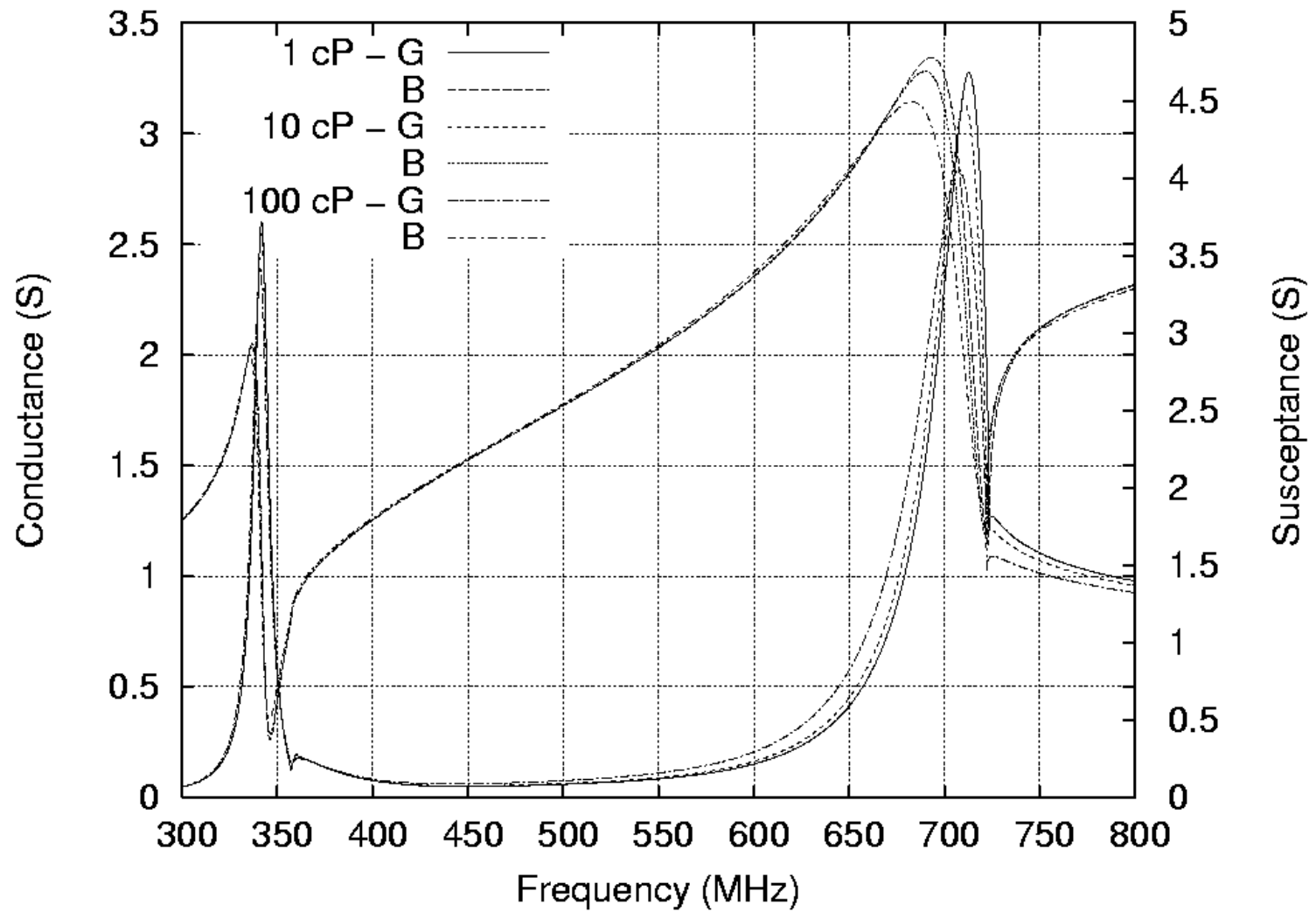

Fig.8 


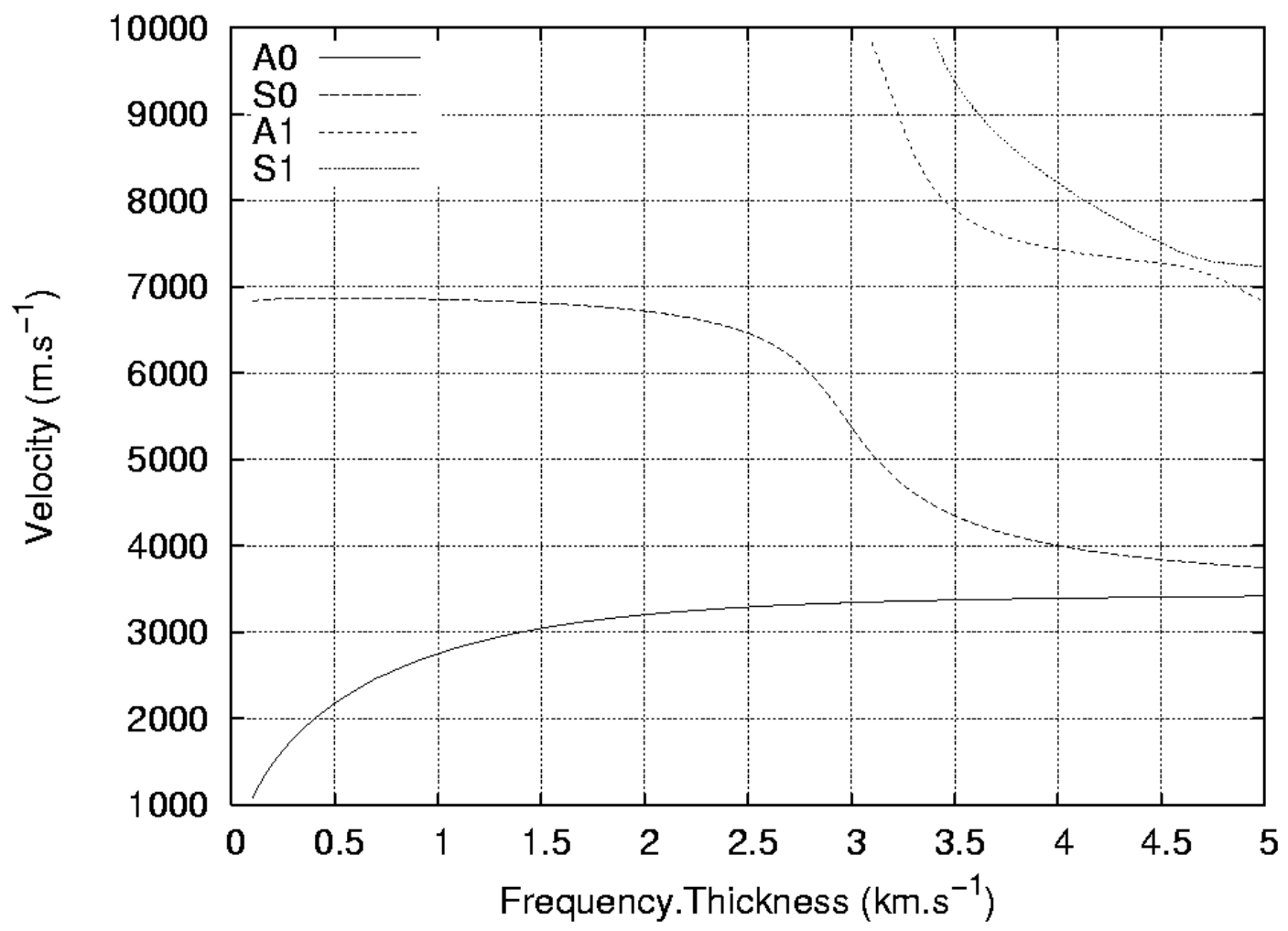

Fig.9 


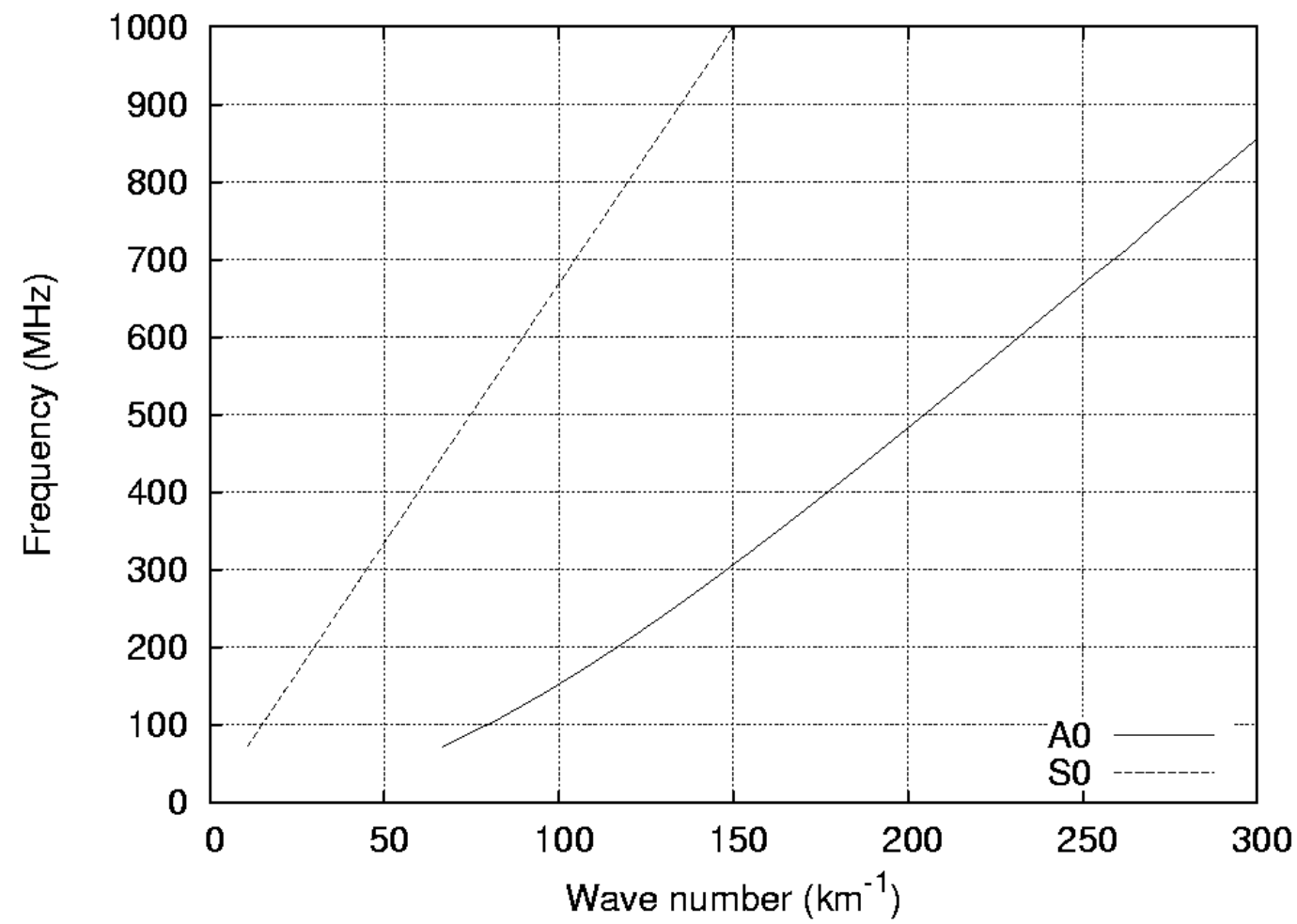

(a)

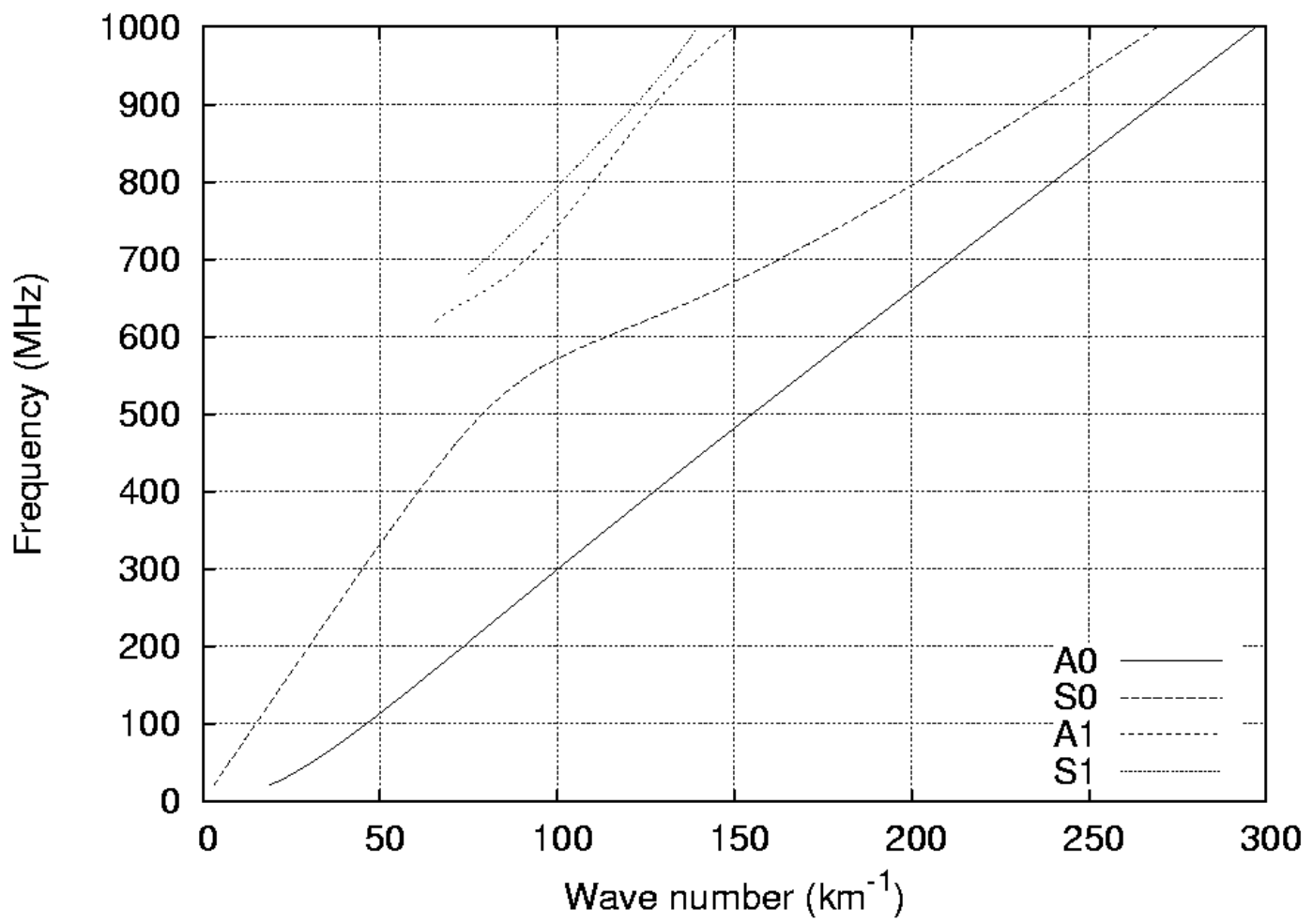

(b)

Fig.10 


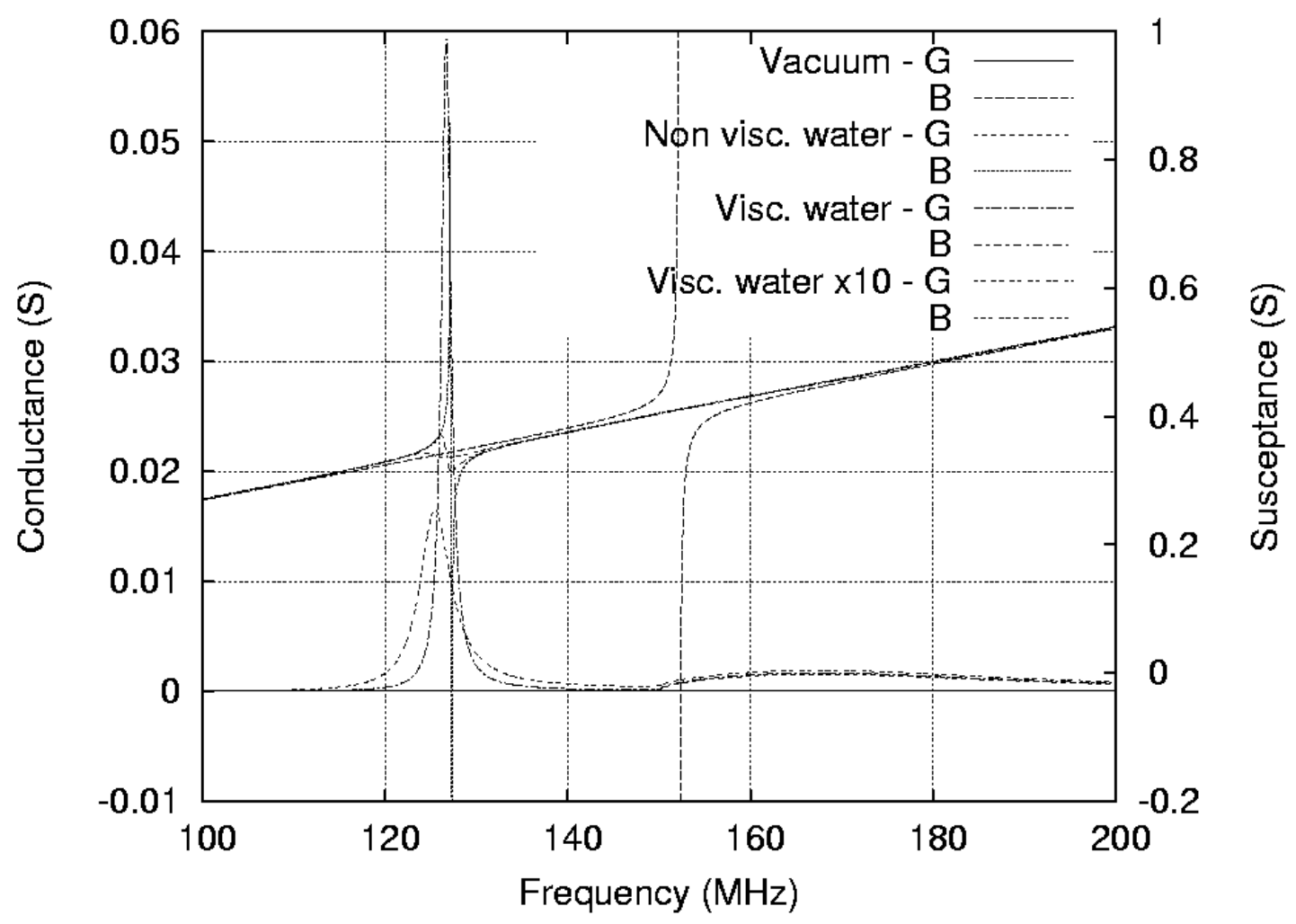

(a)

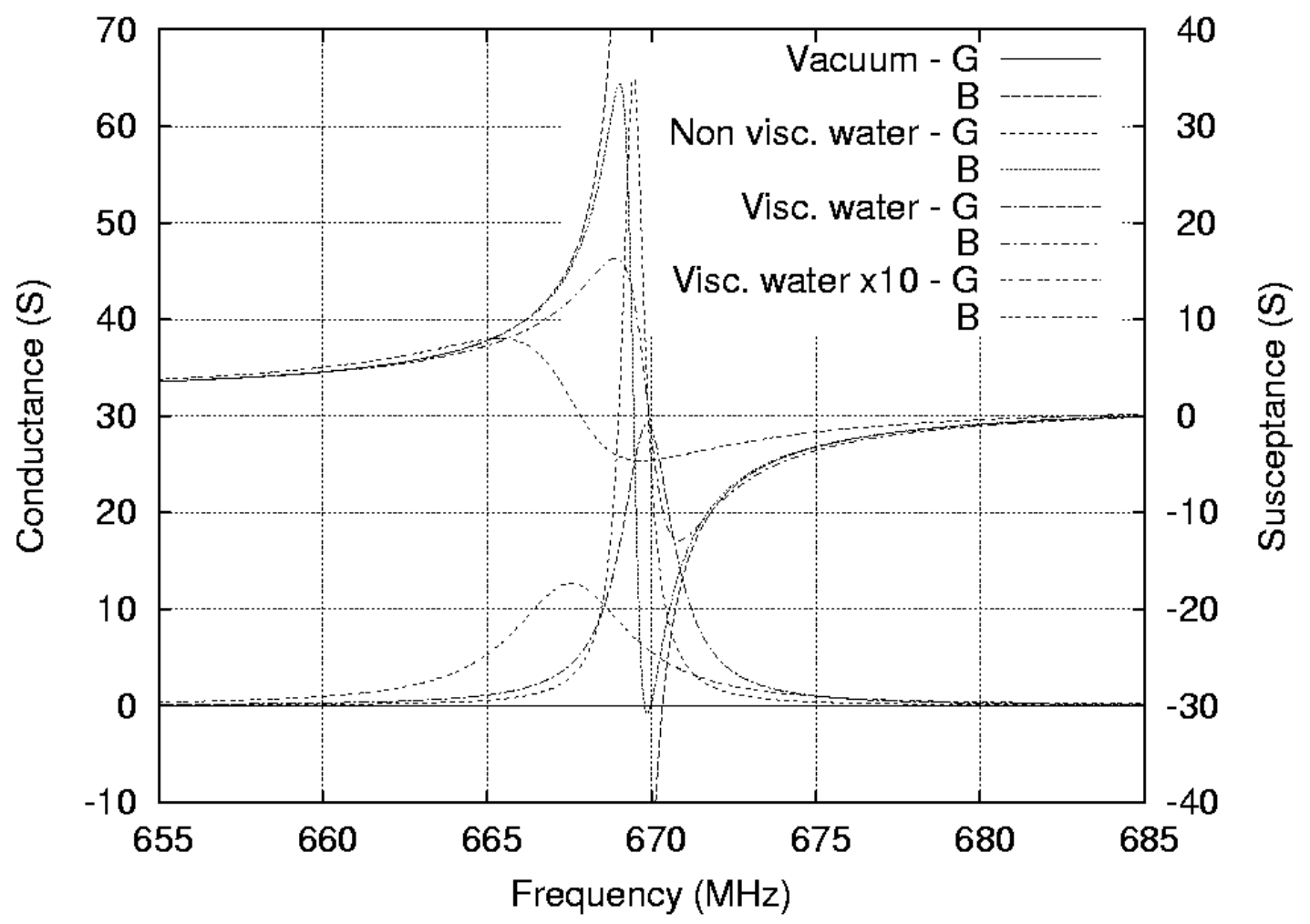

(b)

Fig.11 


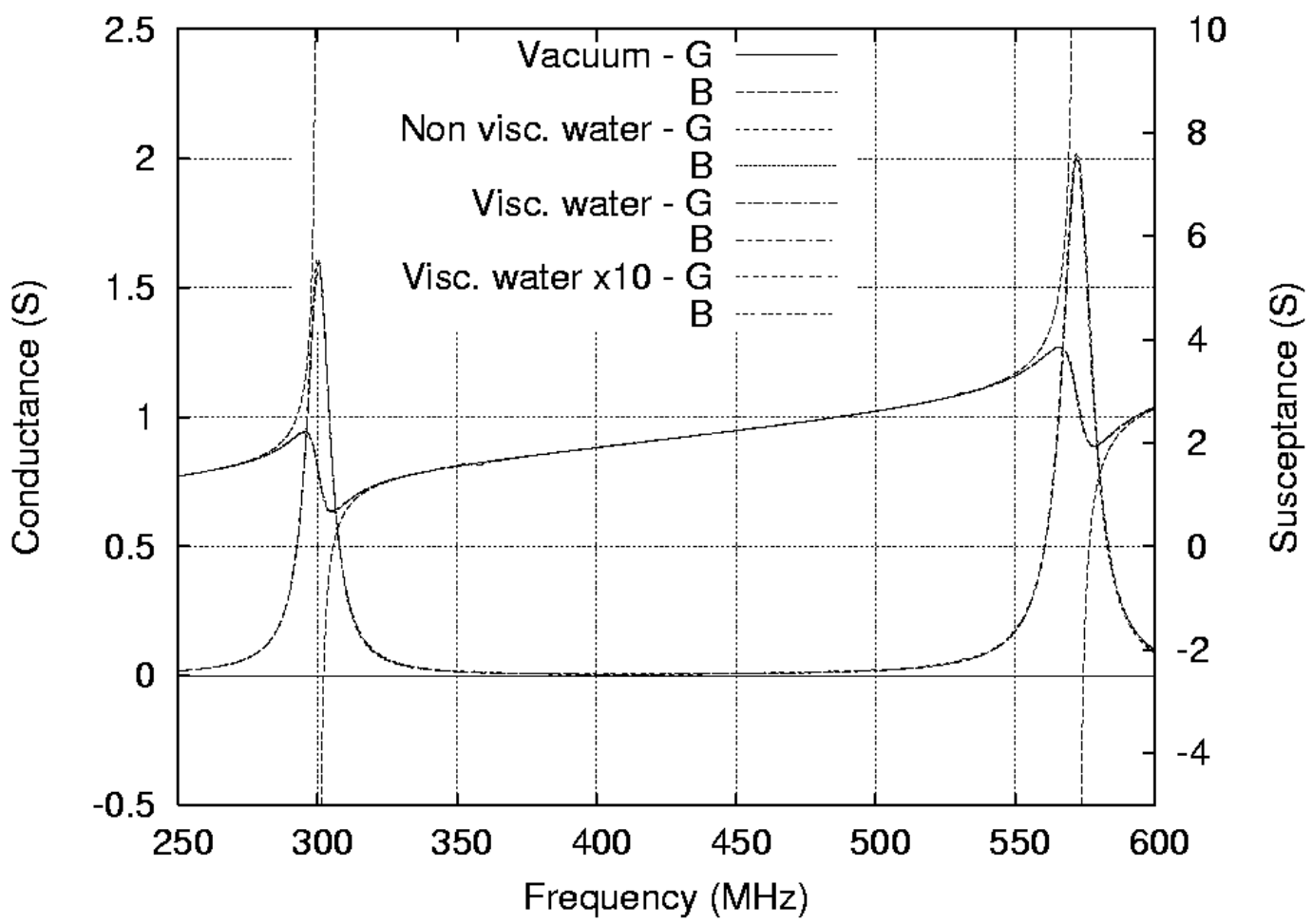

(a)

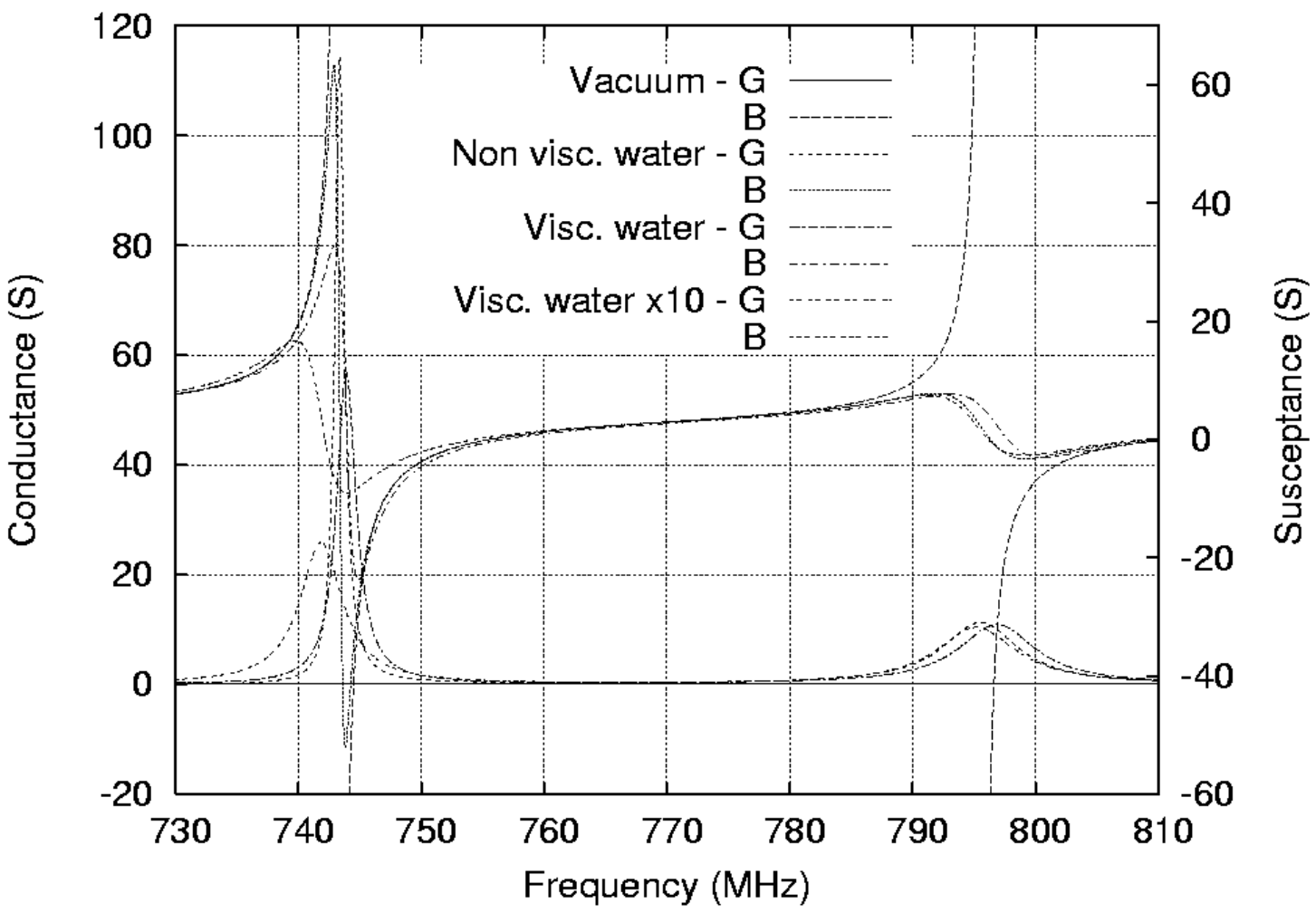

(b)

Fig. 12 